"This is a post-peer-review, pre-copyedit version of an article published in Journal of Signal Processing Systems for Signal, Image, and Video Technology. The final authenticated version is available online at: https://doi.org/10.1007/s11265-0181343-1"

This version is subjected to Springer Nature terms for reuse that can be found at: https://www.springer.com/gp/openaccess/authors-rights/aam-terms-v1 


\title{
Extending the Lifetime of Nano-Blimps via Dynamic Motor Control
}

\author{
Daniele Palossi ${ }^{1}$ • Andres Gomez ${ }^{1,2}$ - Stefan Draskovic ${ }^{2}$ • Andrea \\ Marongiu $^{1,3}$. Lothar Thiele ${ }^{2}$ - Luca Benini ${ }^{1,4}$
}

DOI: https://doi.org/10.1007/s11265-018-1343-1

\begin{abstract}
Nano-sized unmanned aerial vehicles (UAVs), e.g. quadcopters, have received significant attention in recent years. Although their capabilities have grown, they continue to have very limited flight times, tens of minutes at most. The main constraints are the battery's energy density and the engine power required for flight. In this work, we present a nano-sized blimp platform, consisting of a helium balloon and a rotorcraft. Thanks to the lift provided by helium, the blimp requires relatively little energy to remain at a stable altitude. This lift, however, decreases with time as the balloon inevitably deflates requiring additional control mechanisms to keep the desired altitude. We study how
\end{abstract}

This work has been funded by projects EC H2020 HERCULES (688860), Nano-Tera.ch YINS, and Transient Computing Systems (SNF grant 157048).

Daniele Palossi · E-mail: daniele.palossi@iis.ee.ethz.ch

Andres Gomez · E-mail: andres.gomez@iis.ee.ethz.ch

Stefan Draskovic·E-mail: stefan.draskovic@tik.ee.ethz.ch

Andrea Marongiu · E-mail: a.marongiu@unibo.it

Lothar Thiele·E-mail: lothar.thiele@tik.ee.ethz.ch

Luca Benini · E-mail: luca.benini@iis.ee.ethz.ch

${ }^{1}$ Integrated Systems Laboratory (IIS), ETH Zürich, Zürich, Switzerland

${ }^{2}$ Computer Engineering and Networks Laboratory (TIK), ETH Zürich, Zürich, Switzerland

${ }^{3}$ Department of Computer Science and Engineering (DISI), University of Bologna, Bologna, Italy

${ }^{4}$ Department of Electrical, Electronic and Information Engineering (DEI), University of Bologna, Bologna, Italy duty-cycling high power actuators can further reduce the average energy requirements for hovering. With the addition of a solar panel, it is even feasible to sustain tens or hundreds of flight hours in modest lighting conditions. Furthermore, we study how a balloon's deflation rate affects the blimp's energy budget and lifetime. A functioning 68-gram prototype was thoroughly characterized and its lifetime was measured under different harvesting conditions and different power management strategies. Both our system model and the experimental results indicate our proposed platform requires less than $200 \mathrm{~mW}$ to hover indefinitely with an ideal balloon. With a non-ideal balloon the maximum lifetime of $\sim 400 h$ is bounded by the rotor's maximum thrust. This represents, to the best of our knowledge, the first nano-size UAV for long term hovering with low power requirements.

Keywords Self sustainability · Energy neutrality · $\mathrm{UAV} \cdot$ Blimp

\section{Introduction}

The popularity of nano-size unmanned aerial vehicles (nUAVs), typically weighing $\sim 50$ grams, has increased significantly in the past few years $[30,31]$. These UAVs are used for aerial mapping, photography, surveillance, sport, entertainment and others. Despite significant research effort in past years, nano-size UAVs still have short flight times, typically tens of minutes. This has limited their applicability, since longer missions require additional infrastructure to replenish them at service stations $[15,40]$.

A nUAV with long flight times could have a number of innovative applications in surveillance, smart buildings, agriculture, both indoor and outdoor, to name a 
few. Even extending nUAV flight times to only a few days, which is already significantly longer than existing systems, would enable them to collect, process and transmit information from on-board sensors for large areas, as required by many practical applications.

The reduced flight times of existing UAVs are mostly due to the power required for the rotors to generate enough thrust. Even for nUAVs, which typically weigh $\sim 50 \mathrm{~g}$, around $5 \mathrm{~W}$ of power are needed for the mechanical system [7]. This does not even account for the computational requirements implied when dealing with autonomous systems, which adopt power-hungry sensor fusion and real-time control for on-line path planning and collision detection/avoidance algorithms [28]. Given the current battery densities of $500 \mathrm{~J} / \mathrm{g}$ and their limited technology scaling, designing nano-UAVs with flight times of days or weeks will require novel methodologies that combine both hardware and software.

Energy harvesting has been successfully demonstrated in a number of UAV platforms as a way to extend their flight times [19,3]. Photovoltaic cells are a common form of harvester due to their high power density [5] and the general availability of light without specialized infrastructure requirements. But harvested energy is only one side of the equation, and to really maximize a UAV's lifetime, its power requirements must be minimized as well. For many years, power management techniques like duty-cycling have been successfully deployed in battery-based cyber-physical systems in order to reduce the average power consumption and consequently extend the battery lifetime $[4,13]$. Traditional nano-UAVs, however, are fundamentally incompatible with duty-cycling. If a quadrotor tried to shut down its rotors, it will either crash very quickly or incur a significant energy penalty to counteract the acceleration due to gravity.

Fortunately, another type of UAV has certain properties which make it compatible with duty-cycling. A nano-size blimp is a perfect candidate for long flight times because helium, a lighter-than-air gas, can provide lift and significantly reduce the energy requirements for flight. Even though helium provides lift, a perfect balance with a blimp's weight is impossible since even the smallest difference between the system's weight and its lift will result in vertical movement [8]. Even if a system is perfectly tuned at one point, a non-ideal balloon will deflate and eventually reduce the resulting lift to the point where the blimp will not be able to hover (i.e., that is able to maintain a desired altitude within a given tolerance range). The lifetimes of non-ideal blimps are effectively bounded not only by the available energy but also the enclosed helium. Designing a hovering blimp that is able to reach these limits remains a challenge to this day. Though hovering is a one dimensional problem, it is a fundamental building block for the development of fully autonomous UAVs with extended flight times.

In our previous work [29], we explored the energy requirements of blimps with ideal balloons that could hover indefinitely. In addition, we studied how different power management techniques, namely continuous power and duty-cycling, differed in terms of their service level and power requirements. The former can achieve hovering with a relatively small deviation from the desired altitude, at the price of higher power consumption. The latter reduces the average power and leads to longer flight times, but demands a larger tolerance of the desired altitude. In this work, we explore how a real balloon (non-ideal) affects the energy requirements and propose dynamic power management (i.e., DPM) for long-term hovering in presence of deflation. Furthermore, we analyze how different rotor, blade and microcontroller configurations influence the blimp's mechanical efficiency, and thus its power consumption. Finally, we explore how different types of photovoltaic panels and their power output varies in different lighting conditions. Of special interest is the required luminosity level to extend the lifetime of the nano-blimp, given its strongly limited payload. Our proposed platform, consisting of a single rotor controlled by a low-power MCU and a $\sim 0.08 \mathrm{~m}^{3}$ helium balloon, weighs a total of $68 \mathrm{~g}$ and is able to hover for tens to hundreds of hours, requiring only commercial-off-the-shelf components and modest light conditions.

The main contributions of our work are:

- A system model capable of predicting an energy harvesting blimp's lifetime given probabilistic harvesting conditions, solar panel size, and battery capacity.

- An optimization formulation for distributing a blimp's payload, thus determining the battery to solar panel weight ratio which maximizes the blimp's lifetime.

- A study of two types of hovering mechanisms: continuous and duty-cycling, exhibiting a trade-off between energy requirements and hovering precision also in the non-ideal scenario of balloon deflation.

- A characterization of a typical balloon's deflation rate and its effect on the blimp's lifetime.

- A thorough evaluation and model comparison of our $68 \mathrm{~g}$ blimp prototype. Thanks to its power management, a blimp with an ideal balloon would require only $184 \mathrm{~mW}$ of input power for self-sustainable hovering as opposed to $517 \mathrm{~mW}$ needed for continuous operation of the rotor.

- We evaluate a dynamic mechanism to compensate the deflation of a non-ideal balloon, resulting in a total blimp lifetime up to $\sim 400 h$. 
The remainder of this paper is organized as follows: in the next section, we discuss a general classification of UAVs with different sizes and aerodynamics, as well as existing solutions that integrate energy harvesting. In Section 3, we discuss the preliminary overview of hovering, dynamic power management and self-sustainability. In Section 4, we present our system model with probabilistic energy harvesting, and lifetime estimation. In Section 5, we discuss in detail the implementation of our nano-blimp prototype. In Section 6 , we characterize our prototype and evaluate hovering with and without power management. Finally, we conclude our work in Section 7.

\section{Related Work}

Unmanned aerial vehicles (UAVs) with solar energy harvesting have been studied for many years. UAVs, however, can be classified according to different criteria. Each UAV class has its own challenges and limitations, which are tied to the existing technologies in terms of mechanical propulsion, material science, and electrical engineering.

\subsection{UAV Classification}

Rotorcrafts can be classified on the basis of their sizes and power consumption, as reported in Table 1. For the sake of generality, the size refers to the core frame of the vehicle and not the inflatable parts, like balloons in the case of blimps. An additional classification parameter is the vehicle's sensitivity to environmental conditions (e.g. wind, temperature, pressure, etc.), which depends on the vehicle's dimension and speed range. The blimp presented in this paper is considered a nano-UAV due to its low power consumption of $\sim 500 \mathrm{~mW}$, limited payload of $69 \mathrm{~g}$, and small frame measuring about $4 \times$ $4 \mathrm{~cm}$.

\begin{tabular}{llll}
\hline $\begin{array}{l}\text { Vehicle } \\
\text { Class }\end{array}$ & $\begin{array}{l}\oslash: \text { Weight } \\
{[\mathrm{cm}: \mathrm{Kg}]}\end{array}$ & $\begin{array}{l}\text { Power } \\
{[\mathrm{W}]}\end{array}$ & $\begin{array}{l}\text { On-board } \\
\text { Device }\end{array}$ \\
\hline std-size $[21]$ & $\sim 50: \geq 1$ & $\geq 100$ & Desktop \\
\hline micro-size[11] $\sim 25: \sim 0.5$ & $\sim 50$ & Embedded \\
\hline nano-size[7] $\sim 10: \sim 0.05$ & $\sim 5$ & $\mathrm{MCU}$ \\
\hline pico-size $[39]$ & $\sim 2: \sim 0.005$ & $\sim 0.1$ & $\mathrm{ULP}$ \\
\hline
\end{tabular}

Table 1: Rotorcraft UAVs classification by vehicle classsize.
A second dimension for classification is the type of unmanned aerial vehicles: fixed wing, rotorcraft, and blimps. The main trade-offs between the aforementioned types are their maneuverability/controllability and their energy requirements. As depicted in Figure 1-A, a traditional criterion to classify UAVs is given by the trade-off between maneuverability and endurance [35]. In this work we use the concept of agility, as shown in Figure 1-B, defined as: the minimum space required by the vehicle to accomplish a given maneuver, at the minimum speed allowed for the vehicle. Under such definition blimps are more agile than fixed wing vehicles, since they can perform sharp turns within a limited space at reduced speeds. This notion of agility is particularly relevant for indoor applications, where human safety is an important factor. Moreover, the shape and dimension of the balloon compared to the core frame, makes the blimp suitable to perform missions in close vicinity to people and objects. In fact the blimp not only has less moving mechanical parts than a quadcopter but also a lower momentum (due to a reduced velocity). In contrast, blimps are more sensitive to environmental conditions than other UAV types, especially in outdoor scenarios.

Rotorcraft These vehicles have one or more rotors and can achieve stable hovering and precise flight by adjusting rotor speed and balancing different forces. Rotorcrafts are highly maneuverable, can operate in a wide speed range and can take-off and land vertically. They also have very high energy consumption since they need to generate propulsion continuously. The most common rotorcraft is the quadrotor, that has four rotors and changes the rotation ratio among them to generate lift [11].

Fixed Wing These aircrafts, also called airplanes, use fixed wings to generate lift for flight. The shape of the wing pushes air over the top of the wing to flow more rapidly than underneath it, causing a difference in pressure and generating lift [27]. Though fixed-wing UAVs have lower energy requirements and longer flight times compared to quadrotors, they cannot hover or make tight turns, which can limit their deployment in certain applications. In recent years, a new hybrid category has received particular attention: convertibles UAVs [37]. They combine rotorcraft for take-off and landing maneuvers with fixed wing for energy-efficient long-range flights.

Blimps These vehicles, also called airships, have close to neutral buoyancy and can be steered and propelled through the air using one or more propellers [23]. Contrary to other types of UAVs, they can hover thanks to the lift generated by a lighter-than-air gas, and thus require relatively little energy for movement at 

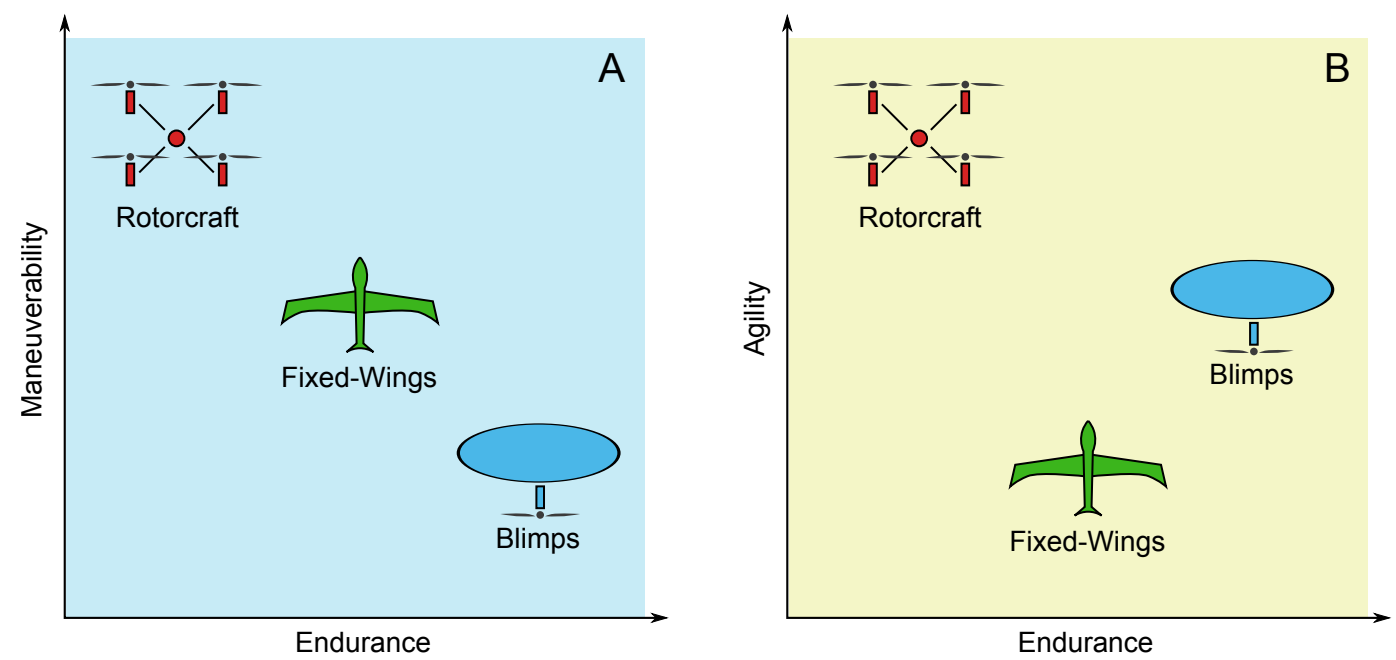

Fig. 1: Classification of UAVs based on their endurance vs. maneuverability (A) and endurance vs. agility (B).

low speeds. Due to their reduced energy requirements, level of agility and sensitivity to the environment, we argue that nano-size blimps are very suitable candidates for indoor application scenarios.

\subsection{Energy Harvesting UAVs}

Despite the challenges associated with high power consumption in quadrotors, researchers have been able to design solar-powered versions. The solarcopter, proposed in [34], uses a $0.96 \mathrm{~m}^{2}$ monocrystalline solar panel, generating $136.8 \mathrm{~W}$ in favorable lighting conditions. A specially designed frame with a high stress resistance to weight ratio was required for the $925 \mathrm{~g}$ quadrotor to fly. Due to its lack of energy storage, this design has flight times limited to periods of high energy availability. One alternative energy source for UAVs with the potential for ultra long lifetimes are laser power beams $[25,26]$. By using a special laser power supply, a ground station can wirelessly direct power to a moving UAV. The authors of [3] present a $1 \mathrm{Kg}$ quadcopter prototype that was able to fly for 12.45 hours powered by laser beams. Such lasers have the benefit of enabling optical communication between the UAV and base station. However, this class of systems requires line-of-sight and additional expensive infrastructure which is not feasible in many applications scenarios. To have a coverage comparable to traditional lighting infrastructures, a laser-based system would require a large number of transmitters, making it even costlier.

Automated battery swap [36] or recharge stations for UAVs [9] have also been studied. In this scenario, the UAV is expected to arrive to a base station periodically, where its battery is restored. This process lasts at least
15 to 30 seconds for battery replacement. Even though the above cited work focuses on quadrotors, automated docking of a nano-size blimp has been shown to be possible as well. For example, in [41] the authors present a small-size indoor blimp that can dock to a station based on visual input. However, apart from the additional infrastructure needed, we note that docking and recharging the blimp would constitute an interruption of the mission.

Fixed-wing UAVs have also been equipped with solar panels to harvest energy during the day. In [18], for example, the SkySailor airplane was able to fly 27 hours during summertime with a wingspan of $3.2 \mathrm{~m}$ using solar panels. Sunsailor [38] achieved a three day flight using a $4.2 \mathrm{~m}$ wingspan and weighing $\sim 3.6 \mathrm{Kg}$. The Helios prototype [24] was developed by NASA for high altitude and long endurance flights. With a $75 \mathrm{~m}$ wingspan and a gross weight of up to $930 \mathrm{Kg}$, it was able to prove sustainable in the stratosphere [19]. AtlantikSolar [27] is a $5.6 \mathrm{~m}$-wingspan, $6.8 \mathrm{Kg}$ of weight, solar-powered lowaltitude long-endurance UAV capable of a continuous flight of 81.5 hours, covering a total of $2316 \mathrm{Km}$. These works demonstrate that standard (and large) size fixed wing airplanes are able to harvest enough energy for long flight times. Nonetheless, it is also understood that these systems must have a scale large enough for the required energy storage systems and propulsion. In addition, these systems suffer from the same limitations: the inability to hover and perform sharp turns due to the minimum high speed required to operate.

For those indoor environments which offer unfavorable conditions for commonly used energy harvesting systems, other techniques known as indoor energy harvesting can be applied. This approach is known as wireless energy transfer. The laser power supply mentioned 
earlier is an example of this, where focused light is used to supply a UAV with energy. Apart from lasers, it has been shown that diffused light, magnetic coupling, or radio frequency transmissions offer the possibility of transferring energy beyond the centimeter scale as well [5]. Using magnetic coupling, $60 \mathrm{~W}$ of energy were experimentally transferred over $2 m$ with a $40 \%$ efficiency [17]. The coils used in that case were $30 \mathrm{~cm}$ in diameter with a $20 \mathrm{~cm}$ height, thus a coil for harvesting could be fitted on a blimp. Using radio frequency energy can be transmitted in the kilometer range, though similarly to the solution based on lasers, line-of-sight is required. For example, the $P 1110 B$ Powerharvester receiver [2] can harvest hundreds of milliwatts within a short range. For both these cases additional costly infrastructure is needed to send the energy for the wireless transfer.

Solar-powered blimps offer the best-case scenario for UAVs requiring ultra long flight times, due to their reduced energy requirements. In [20] the trade-offs between solar panel weight and power produced are chosen for a high altitude blimp and validated using design parameters from $[22,16]$. In [12], the effect of the curvature of the balloon surface and the corresponding changes in the energy output is analyzed for a solar powered lighterthan-air UAV platform. All of these studies focus on large solar-powered, lighter-than-air blimps, since they are required to withstand adverse weather conditions for prolonged periods of time, particularly those meant to operate in the stratosphere. These blimps are up to $400 \mathrm{~m}$ in length, and consume $100 \mathrm{KW}$. In the case of [12], the blimp has a volume of $24 \mathrm{~m}^{3}$ and requires only $100 W$ of power.

Our work focuses on a nano-scale blimp with a weight of less than $70 \mathrm{~g}$, a non-ideal balloon of $\sim 0.08 \mathrm{~m}^{3}$ and a single core-less rotor. The proposed DPM allows the nano-blimp to reach a maximum lifetime of $\sim 400$ hours. To the best of our knowledge, this work presents the first nano-size UAV capable of continuous, long term hovering. Thanks to its energy harvesting capability and the low deflation rates of balloons with low permeabilitymaterials (e.g., mylar), the blimp platform presented in this paper could conceivably hover for several weeks in indoor environments.

\section{Building Blocks for Long-Term, Nano-UAVs (nUAVs)}

Though there is increasing interest in autonomous and intelligent aerial vehicles, most of the existing devices are either very large systems, or have flight times in the order of minutes. Since battery densities are still far below the requirements for nUAVs, we believe the following elements to be essential for long-term nUAVs: compatible rotor craft, power management and energy harvesting. First, the rotor craft has to tolerate variable propulsion without excessive overhead. If reducing the rotor's intensity invariably leads to a crash of the vehicle, there is no way to save energy through software alone. Second, power management techniques are necessary to reduce power consumption while taking into account environmental and physical constraints. Lastly, harvesting can significantly extend the battery's lifetime if the environmental conditions are favorable. To demonstrate how these concepts can lead to long-term, functional nano-sized blimps, we will study the problem of hovering. Though hovering is the simplest action a nUAV can perform, it still requires significant energy for nUAV flight and can give insights to the more complicated problem of free movement in $\mathbb{R}^{3}$.

\subsection{Rotor Craft Setup}

The blimp's rotorcraft is built using a modified, opensource/open-hardware nano-quadcopter, the Crazyflie 2. $0^{1}$. The quadcopter originally weighed $26 \mathrm{~g}$ with the battery and flies for approximately 15 minutes per charge, in standard conditions. This craft was chosen due to the form factor and the open source design allowing flexible software and hardware modifications.

The drone's original hardware is built around two MCUs, a collection of sensors, and four motors providing the lift. The frame of the craft is the circuit board itself and the motors are attached to the PCB using plastic motor mounts. The radio communication and power management for the system is controlled via a NRF51 $M C U^{2}$. The motors are controlled by an ST STM32F405 $M C U^{3}$ by pulse-width modulation (PWM) signals.

The craft was modified to provide lift to support the goal of hovering. Figure 2-A shows the final design of the prototype. Only one rotor is attached to the craft and is pointed downward to provide upward thrust to the blimp. The single rotor was mounted in the center of mass, otherwise an oscillating movement would have been generated, compromising the stability of the system. The blade is selected in order to have the desired thrust in the right direction. This can be achieved combining the clockwise $(\mathrm{CW})$ rotor with counter clockwise (CCW) blade mounted backwards, as depicted in Figure 2-B. The system is extended with the tiny TI bq2920 4 power charger to convert the energy harvested from the solar

\footnotetext{
1 http://www.bitcrincludingaze.io/crazyflie-2

2 http://www.nordicsemi.com/Products/nRF51-Series$\mathrm{SoC}$

3 http://www.st.com/en/microcontrollers/stm32f405-415

4 http://www.ti.com/lit/gpn/bq29200
} 


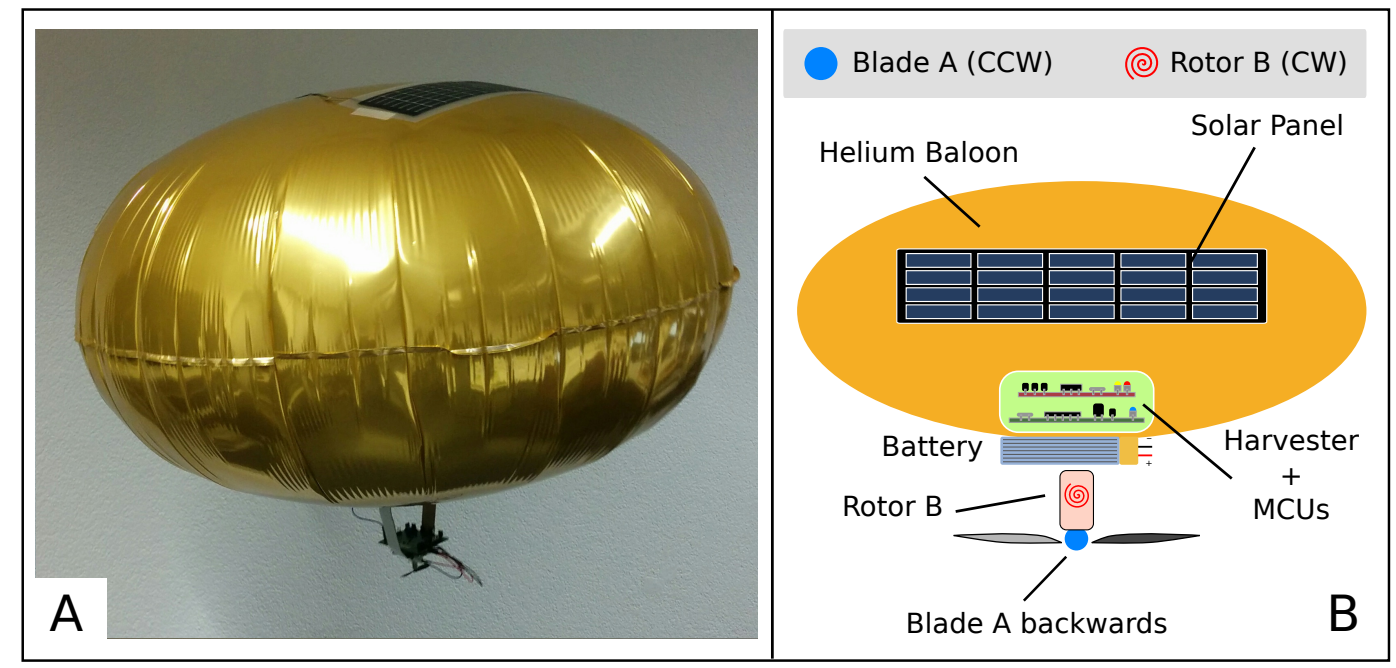

Fig. 2: A: The blimp prototype during flight. B: The blimp model with solar panel, MCUs, battery, and rotor.

panel. Finally, all the hardware components are attached to the underside of the balloon using a lightweight frame.

The solar panel is mounted on top of the balloon and the rotorcraft is suspended from the balloon's underside. This setup can be seen in Figure 2. The suspended rotorcraft requires a stiff harness to avoid swinging during flight and acting as a pendulum. The helium balloon used for the blimp is a commercially available, ellipsoidal $60 \times 65 \times 40 \mathrm{~cm}$ mylar balloon. Mylar is a commonly used material, which is sturdier than the common latex balloon and has a lower gas permeability that allows balloons to stay inflated longer.

The balloon's maximum lift was determined experimentally to be $\sim 69 \mathrm{~g}$. All of the wires, battery, rotor, solar panel, and hardware needs to fit under that weight budget. Our modified rotorcraft weighs $10 \mathrm{~g}$, the additional connections accounts for $3 g$, and the power converter board for $16 \mathrm{~g}$, thus the available payload left for both battery and solar panel is $40 \mathrm{~g}$. In Section 6.1 we will evaluate the change in lifetime under different light conditions and battery/solar panel weights.

\subsection{Hovering under Static Conditions}

One of the most basic tasks that non-airplane UAVs need to perform is hovering, which keeps the aircraft at a stable altitude. Figure 3 shows a basic comparison between a hovering quadrotor and a blimp. The quadrotor needs to continuously generate thrust from its four rotors to be able to counteract gravity. This results in an enormous power requirement. A blimp, on the contrary, leverages a lighter-than-air gas like helium to generate lift passively. This significantly reduces the energy re- quirements since relatively little thrust is necessary to counteract gravity.

Though in theory a blimp can passively hover with neutral buoyancy, this is very hard to achieve in practice. This would require perfectly calibrated weights to offset a balloon's lift in a given environment. Any small change to the environmental conditions (e.g. temperature, pressure, humidity, etc.) would affect the balloon and its steady-state altitude. But even in a controlled environment, the balloon's deflation rate will quickly result in slightly negative buoyancy, which will eventually drive the balloon to the ground. For a balloon to hover long-term at a desired altitude, active control is required. The focus of this paper is to reduce the power requirements of hovering in controlled environments to maximize the balloon's lifetime.

Our proposed blimp platform will be a slightlyheavier-than-air system, such that it falls slowly and requires relatively little energy to achieve, on average, neutral buoyancy. ${ }^{5}$ We want to go even further by borrowing power management concepts commonly found in digital systems and implementing them in a nanoblimp. Duty-cycling is a technique in which a system periodically transitions from a power-hungry on state to a low-power off state. Depending on the ratio between on and off times, the system's average performance and power consumption will vary. While there typically are transitions overheads associated with duty-cycling, they can be compensated if it puts the system in a more efficient operating point. For our particular blimp system, duty-cycling the rotors at their highest intensity will

5 Note that, a platform slightly lighter-than-air, coupled with a propeller generating lowering would be feasible as well. However, with this design the deflation of the balloon over time would require a second rotor generating lift. 


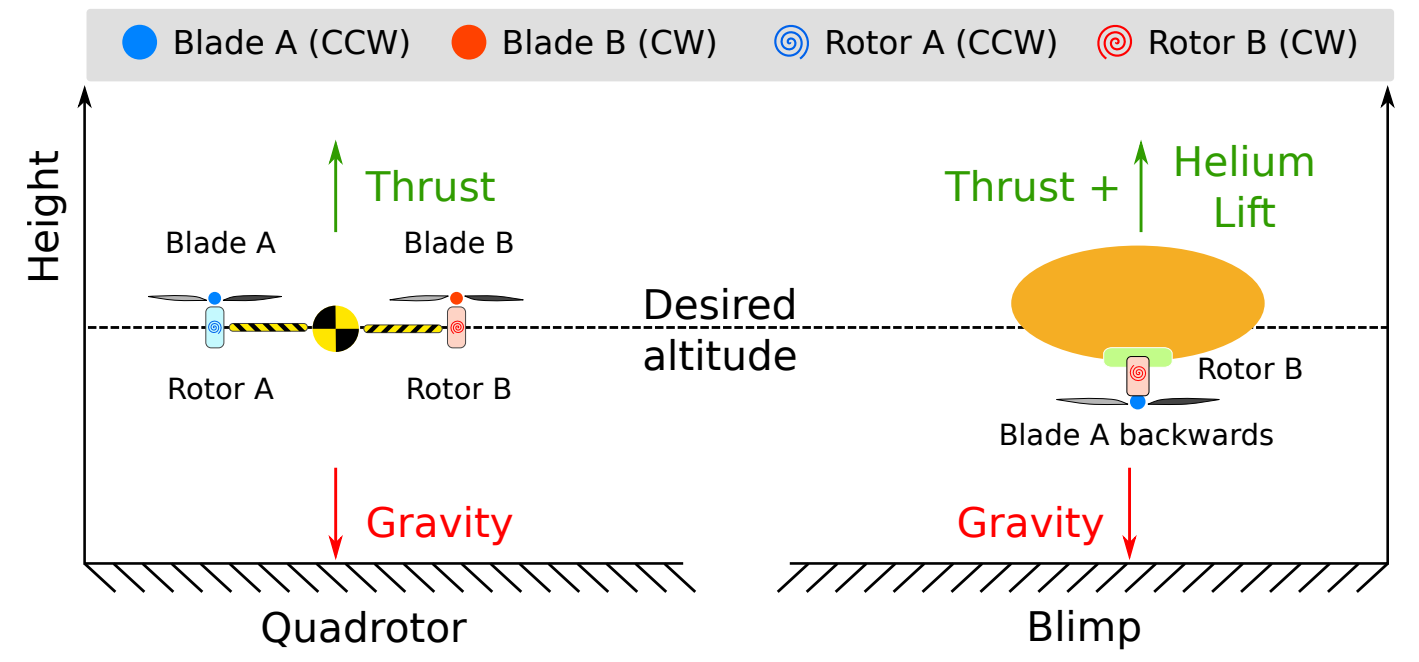

Fig. 3: For a quadcopter to hover, active thrust is necessary to compensate the weight. A blimp requires significantly less thrust due to the lift generated by helium.

be more efficient than operating them at a reduced and constant intensity.

Figure 4 shows two hovering methods for blimps in controlled environments. On the left, a continuously powered, low-intensity rotor can maintain a stable altitude. With an ideal balloon, the lift will be constant and the rotor can offset gravity with a continuous (and constant) intensity/power. On the right, duty-cycling a high-intensity rotor can achieve, on average, the desired altitude. Even though duty-cycling requires a higher rotor intensity and has an additional overhead compared to a continuously powered rotor, it can still reduce the overall energy because the system is more efficient when the motors operate at a higher intensity. This is due to power conversion circuity, whose efficiency typically improves at higher voltage/currents. Since the selected rotors are voltage controlled, low intensity means low voltage and thus results in a lower conversion efficiency. For this reason we duty-cycle with the maximum intensity, thus minimizing losses. Increasing the $t_{\text {on }}$ also makes each activation cheaper, since the initial start-up peaks will be amortized over a longer active time. Con- a) Continuous Rotor
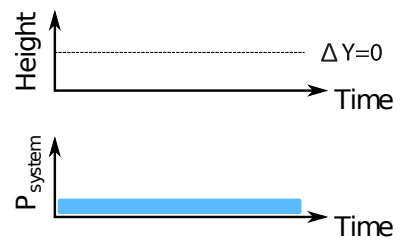

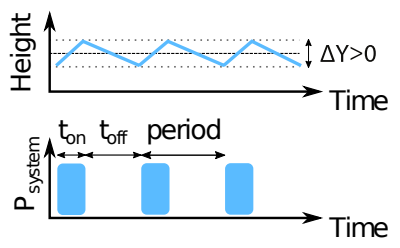

Fig. 4: Assuming an ideal balloon, blimps can hover indefinitely using two statically defined techniques: continuously powered and duty-cycled rotors. versely, the $t_{o f f}$ and the $\Delta \mathrm{Y}$ will also need to increase, so depending on the mission's tolerance there will be an upper bound on the activation period. If the balloon's lift is constant, the $t_{o n}, t_{o f f}$ parameters can be statically chosen. These techniques, which assume a constant balloon lift, will be referred to as static power management (SPM). Our experiments will show that under SPM, the power savings from duty-cycling substantially extend the blimp's lifetime.

\subsection{Hovering under Dynamic Conditions}

While blimps generate "free" lift from the helium enclosed in the balloon, they can only do so for a finite amount of time. Helium escapes from different balloon materials at different rates, due to their porosity. As the balloon's lift decreases, the resulting acceleration due to gravity will increase, making the blimp fall faster. For this reason, the energy budget grows as the balloon deflates since higher power is needed to counteract gravity. After a long time, the balloon will be so deflated that it will practically generate no lift. This effect clearly has impact on a blimp's long term deployment, effectively placing an upper bound on the lifetime. However, since our rotorcraft has only a single rotor, the blimp will fall to the ground as soon as the blimp's weight exceeds the combined balloon lift and maximum rotor thrust.

The deflation rate of the helium contained in the mylar balloon is measured as the loss of the lift capability, as depicted in Figure 5. If one would start from a perfect buoyancy condition, it would take $\sim 50$ hours before the blimp would fall down with a mean velocity of $0.1 \mathrm{~m} / \mathrm{s}$. In Figure 5, this mean fall down velocity (MFDV) is 


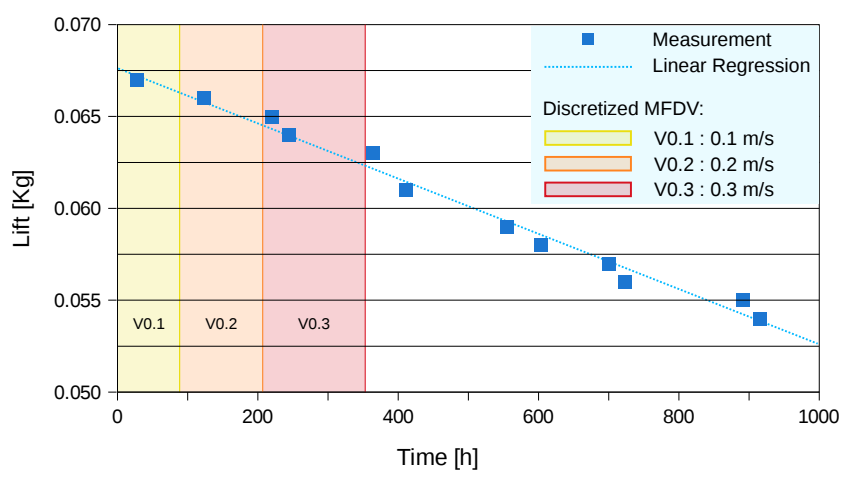

Fig. 5: Loss in lift capability of the mylar balloon over time. In yellow, orange and red the three areas respectively corresponding to a fall down velocity (of the initial 68 grams system) of $0.1,0.2$ and $0.3 \mathrm{~m} / \mathrm{s}$.

represented by the first yellow area on the left (i.e., V0.1). This area represents the time for which the loss of helium results in a MFDV of $0.1 \mathrm{~m} / \mathrm{s}$. Both in Figure 5 and in the rest of our experiments we always consider, as starting time (i.e., 0 on the $\mathrm{x}$-axis), the time at which we have a MFDV of $0.1 \mathrm{~m} / \mathrm{s}$. In Figure 5 we also introduce two other areas in which the MFDV is 0.2 and $0.3 \mathrm{~m} / \mathrm{s}$, respectively named $V 0.2$ and $V 0.3$.

The measurements indicate that the helium loss is linear. A system combining this blimp with a battery, but otherwise incapable of harvesting energy from the environment, would last for $\sim 4$ hours, making the balloon's deflation negligible with respect to the vehicle's lifetime. If we consider, in addition to the battery, the input power harvested from an on-board solar panel, the deflation of the balloon becomes a key aspect to consider when trying to maximize the system's lifetime. Thus, a mechanism able to dynamically adjust the motor thrust will be required to compensate the natural loss of the lift. Our proposed mechanism, called dynamic power management (DPM), is depicted in Figure 6. If applied to a continuously rotor scheme, the rotor intensity will be increased as a function of the deflation, to maintain the desired height. In the case of duty-cycled rotors, the duration of $t_{\text {on }}$ will be adjusted, up to the point where it never turns off. In addition, $t_{\text {off }}$ decreases as the balloon deflates due to the blimp's increased acceleration, resulting in an increased activation frequency to maintain the desired altitude.

\subsection{Energy Harvesting}

As it has been previously discussed, blimps have relatively low energy requirements for hovering. Thanks to our proposed power management techniques, these requirements can be reduced even further. Still, energy har- a) Continuous Rotor

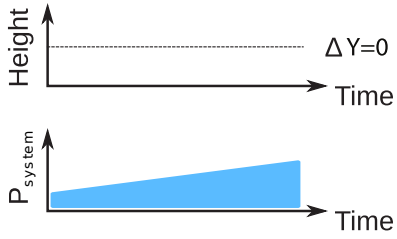

b) Duty-Cycled Rotor

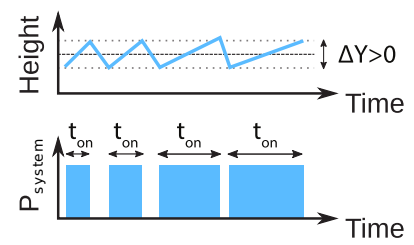

Fig. 6: When the balloon's deflation is taken into account, the hovering algorithm's parameters need to be adjusted dynamically to maintain the desired height. At a certain point, it will no longer be possible to duty-cycle, when its necessary to keep the rotors on at maximum intensity.

vesting is necessary to allow for long-term autonomous operation.

Energy harvesting encompasses a variety of methods to acquire energy from the environment. Solar panels are most common, due to their high power densities and general availability of light. The power produced from a solar panel depends directly on the amount of light and the size of the panel. The first parameter is environmental and cannot usually be controlled. The second parameter is an important design choice. Larger panels can naturally produce more power for a given amount of light, but there is a strict limit since every nano-UAV has a very tight payload. Due to the inherent variability of the energy input, there is a trade-off between the amount of energy a solar panel can harvest and its size/weight.

In energy-harvesting UAVs, where flight times depend on the amount of harvested energy, one of the most relevant parameters is excess time [19]. Excess time measures how long the vehicle is able to fly without any energy input, or simply its minimum guaranteed flight time. This time is provided by batteries, and needs be chosen at design time. The total payload will then depend on the battery to solar panel weight ratio, which can be optimized for a given environmental setting. The payload optimization problem will be studied in greater detail in Section 4.2.

\section{System Model}

In this section we introduce the models and methods for the system's analysis. The first model is used to understand the blimp's power requirements and to estimate the lifetime as a function of environmental conditions. The second model is used to explore the weight distribution problem in order to identify the best trade-off, between dimensions of the battery and the solar panel, for the desired lifetime. 


\subsection{Sustainability Model}

In order to understand how power is harvested and consumed, and to estimate the lifetime of a given blimp, a sustainability model has been created. More precisely, the model we built takes the blimp's configuration and environmental conditions as input, and produces the expected flight time of the craft, where an infinite flight time means the system is self sustainable. By the blimp's configuration, we mean a given solar panel, battery, and a rotor. The rotor's configuration, intensity, power consumption, and the period when duty cycling is used, are included in the configuration as well. To imitate the inherently variable environmental conditions, we describe the harvested energy with a random variable. This implies that the flight time is modeled as a random variable as well.

With this in mind, we have created a discrete time Markov model. Generally speaking, Markov models use states to represent possible conditions the system could be in, while transitions between states are nondeterministic and happen with a certain probability. This means that, at a given time, the probability of the system being in each of the possible states is known.

In our model, the system's state corresponds to energy in the battery available for use. The probability of the transition from one state to another is derived from the consumed and harvested energy in the following way. We first subtract the energy consumed in a period, and then we add the energy harvested in the period. As the battery is finite in size, there are corner cases when the battery is empty and full that need to be taken into account. The time step of this discrete model is one duty-cycle period. There is a computational trade-off between the number of states and the accuracy of the model. However, we found a satisfactory solution by having the difference between two consecutive energy states an order of magnitude smaller than the energies consumed or harvested in one period.

Besides the many states representing different energy levels, there is an additional error state. This state is entered when the rotor fails to run due to insufficient energy. This state is absorbing, meaning that once entered, the system stays in the error state. Because we assume that energy is first consumed during a transition between two states, before being harvested, the whole analysis is pessimistic w.r.t. the error state. By observing the probability of the system being in the error state after running for an amount of time, we can understand whether the blimp is operating at that time or not.

The state representing the battery fully charged is also notable. This is because the battery is finite, and if

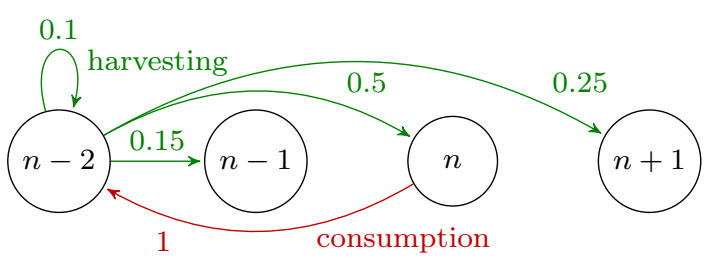

Fig. 7: A transition from $n$ to possible new states

too much energy is harvested, the battery saturates to the fully charged state.

Before formally defining the Markov model, we will present an example to familiarize the reader to the underlying idea.

Example The example in Figure 7 helps to understand the system model. Let the system be in state $n$ at time step $\tau$, meaning its energy level is $n$ at that time. We will determine the state of the system at the next time step $\tau+1$, after one period of operation.

During the period, we assume energy is first consumed and then harvested. Assume that the difference between two energy levels is one energy unit. Let the system consume 2 units of energy per period, and harvest some units of energy per period with the following probabilities: $\left(\begin{array}{cccc}0 & 1 & 2 & 3 \\ 0.1 & 0.15 & 0.5 & 0.25\end{array}\right)$. In Figure 7, the two operations that make up one transition are marked red (consumption) and green (harvesting). We see that the system moves to one of these several states after the period: $\left(\begin{array}{cccc}n-2 & n-1 & n & n+1 \\ 0.1 & 0.15 & 0.5 & 0.25\end{array}\right)$. By continuing this analysis and having more transitions, the possible states the system is in after an arbitrary number of periods can be obtained. Please note that we have not depicted the corner cases in the example: the error state, which is entered when there is not enough energy to supply the rotor; and the full battery state, after which no more energy can be harvested.

Formally, we define the state vector as $(1)$, where $b_{i}^{(\tau)}$ is the probability that the system is in state $i$ at time step $\tau$, and $\sum_{i} b_{i}^{(\tau)}=1$. There are $N$ states representing different energy levels in the battery, and one error state.

$b^{(\tau)}=\left(\begin{array}{lllll}b_{1}^{(\tau)} & b_{2}^{(\tau)} & \ldots & b_{N}^{(\tau)} & b_{\mathrm{err}}^{(\tau)}\end{array}\right)$

We define the transition matrix $T$ as a matrix whose element $T_{i j}$ is the probability that the system transitions from state $i$ to state $j$ in one time step. As the time step of the model is one period, and we assume the consumption of energy happens first, we can write the transition matrix as $T=T_{\text {consume }} \times T_{\text {harvest }}$. Here $T_{\text {consume }}$ represents removing energy from the battery, the amount depending on the energy consumed in a burst; while $T_{\text {harvest }}$ represents adding energy to the 
battery, the amount likewise depending on the energy harvested in one period.

Combining all of the above, we can write the state of the system at time step $\tau$ as $(2)$. Note that $(\tau)$ in the superscript notes a state vector corresponding to time $\tau$, while $\tau$ in the superscript denotes the exponential.

$b^{(\tau)}=b^{(\text {initial })} \times\left(T_{\text {consume }} \times T_{\text {harvest }}\right)^{\tau}$

Finally, we can define the system's lifetime as (3). We see that the system's lifetime is $\tau$ if, for some defined $\epsilon$, the condition is met. If this condition is never met, we say that the system is self sustainable.

lifetime is $\tau \Leftrightarrow$ minimal $\tau$ such that:

$$
b_{\mathrm{err}}^{(\tau)} \leq \epsilon \text { and } b_{\mathrm{err}}^{(\tau+1)}>\epsilon
$$

With the overall structure of the model in place, we can go into more detail regarding the period, battery, and the consumption and harvesting of energy.

\section{Period}

When the rotor operates with duty cycling, the period is the time between two consecutive bursts. In the case of Static Power Management (see Figure 4), this value remains the same throughout the lifetime, however this does not need to be the case with Dynamic Power Management (see Figure 6). Indeed, for practical reasons, some of the prototype DPM configurations feature a period that does not strictly remain the same all the time. However, due to simplicity, the model shall assume power is consumed continuously, i.e. instead of having two output power levels while duty-cycling, we use one average output power. Other behavior, such as the harvesting of energy, is done continuously.

\section{Battery State}

The battery is not a perfect power supply. As the battery voltage level decreases below a certain point, the drone is unable to draw the amount of power required for the rotor. Experimentally, we observed that this level depends on the rotor configuration. The minimum voltage level is lower for continuously powered rotors than it is for duty cycling. We model the battery as a perfect power supply, but its capacity is adjusted such that it reflects the amount of usable energy it can provide to the rotor.

\section{Blimp Power Consumption}

The discharge rate depends on the way the rotor is configured to operate. When configured to continuously power the rotors, the energy consumption per period will be called $E_{\text {const }}$. Eq. (4) shows this energy to be simply the system's constant power consumption times the period.

$$
E_{\text {const }}=P_{\text {const }} \cdot \text { Period }
$$

When duty-cycling, the energy consumption per period, called $E_{d u t y}$, has several parameters. Eq. (5) shows it depends on the power consumption during the on and off periods ( $P_{\text {on }}$ and $P_{\text {off }}$ respectively) and their duration ( $t_{o n}$ and $t_{\text {off }}$ respectively). Note that $t_{o n}+t_{o f f}=$ Period. There is one additional term, $E_{\text {startup }}$, which represents the overhead to turn on the motor in every period. This was omitted in the continuous configuration, since it is incurred only once during the blimps entire lifetime.

$E_{D C}=P_{o n} \cdot t_{o n}+P_{o f f} \cdot t_{o f f}+E_{\text {startup }}$

Another matter is whether the rotor is configured to operate with Static or Dynamic Power Management. On the one hand, when SPM is used, the values $E_{\text {const }}$ and $E_{D C}$ are constant throughout the lifetime, as the motor intensity for continuously powered rotors and the burst duration for duty-cycled operation do not change. This means that each period, the battery state will decrease for a constant amount.

On the other hand, when DPM is used, $E_{\text {and }}$ and $E_{D S}$ become a function of time, as they increase over time as the system operates. As far as the model is concerned any function may be used, and in practice we have found that the output power increases quadratically with the mean fall down velocity (MFDV). This means that energy consumption depends on the how long the system is in operation, and the battery state will decrease each period by a larger amount as time goes by. Note that, in the case DPM is used, the period will also change over time. For battery states that do not have sufficient energy for consumption, the system transitions to the error state.

\section{Probabilistic Energy Harvesting}

By probabilistic energy harvesting, we mean having the probability of adding an amount of energy to the battery during each time period. Note that the probability distribution of energy can vary depending on the environment, and we can analyze arbitrary probability distributions. 

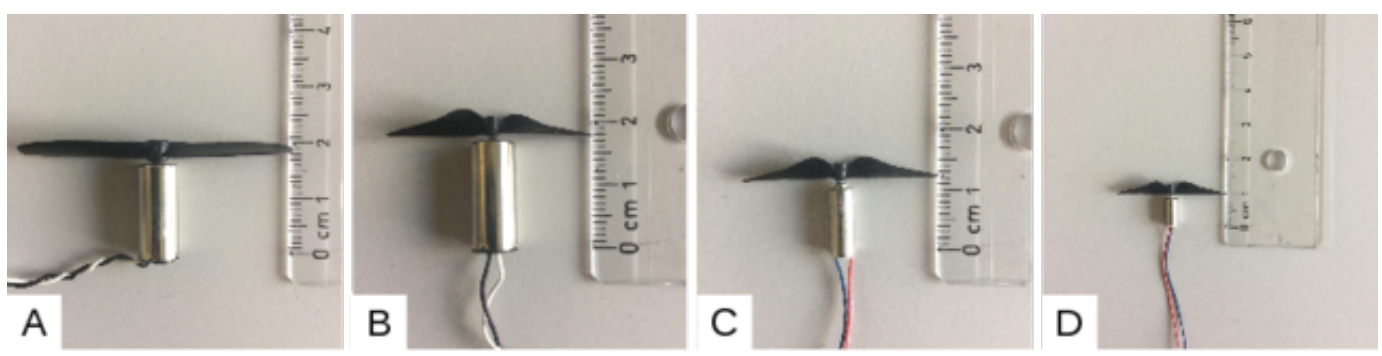

Fig. 8: Motor configurations evaluated, named from left to right Big-B (A), Big-S (B), Medium (C) and Small (D).

This is especially useful for modeling harvesting based on measured data.

\subsection{Dimensioning an Energy Harvesting Blimp}

Every aerial vehicle has a limited payload it can lift. To maximize a blimp's lifetime requires solving a weight distribution problem, where the payload must be optimized to minimize the weight for both solar panel and battery. Thus, a well configured system should be able to harvest and store enough energy for the desired lifetime, saving as much weight as possible. Fundamental parameters to take into account are the target lifetime $(\tau)$ and the illuminance (intensity, variance and duration). Naturally, such parameters depend on the application scenario we want to address, and can vary significantly from one environment to another (e.g. indoor vs. outdoor).

The total weight of the system $\left(W_{t o t}\right)$ is then equal to the sum of each piece: the core frame $\left(W_{\text {frame }}\right)$, the battery $\left(W_{b a t}\right)$, and the solar panel $\left(W_{\text {panel }}\right)$. This total should be less than the maximum payload $\left(W_{\max }\right)$. The average power consumption of the vehicle $\left(P_{\text {load }}\right)$ is supplied from both battery and solar panel. The input energy harvested from the solar panel $\left(E_{i n}\right)$ depends on the panel's area (that is proportional to its weight $\left.W_{\text {panel }}\right)$ and on the illuminance conditions ( Light). The energy supplied by the battery $\left(E_{b a t}\right)$ depends on its weight $W_{b a t}$. Thus we want maximize the lifetime $\tau$, and respect the following conditions:

$$
\begin{aligned}
\tau \cdot P_{\text {load }} & \leq E_{\text {in }}\left(W_{\text {panel }}, \text { Light }\right)+E_{\text {batt }}\left(W_{\text {batt }}\right) \\
W_{\text {tot }} & \leq W_{\max }
\end{aligned}
$$

Our proposed solution, to be discussed in detail in Section 6.1, will evaluate different weight distributions, and estimate the blimp's lifetime for both optimistic and pessimistic lighting conditions.

\section{System Implementation}

In Section 3.1, the main components of the nano-size unmanned aerial vehicle (nUAV) have been introduced. The blimp prototype consists of four main hardware components: the balloon, the solar panel, the battery, and the rotorcraft. Each of these components was carefully selected to optimize the blimp's lifetime. The balloon's dimensions and deflation rate was presented in Section 3.3. The payload distribution problem to size the solar panel and battery was introduced in Section 4.2. In this section we will describe the rotor selection and the software implementation used for both static power management (SPM) and dynamic power management (DPM).

\subsection{Rotor Selection}

The rotor is the most important mechanical component since it generates the thrust necessary to maintain the desired altitude. Since we want to minimize the blimp's total weight, we will only consider a single rotor. To select the rotor, two variables must be fixed: the electrical motor size, and the blade size. It is the combination of both parameters that will determine the mechanical system's power dissipation, generated lift, and consequently its efficiency. In general, larger motor/blade configurations can operate at a higher efficiency than smaller ones, but they do require higher rotational speeds. We explore the power vs efficiency trade-off between different configurations and select the one which maximizes the system's lifetime. The motors/blades evaluated in this work are shown in Figure 8.

The configuration in Figure 8-A (called Big-B) represents the same configuration (motor and blade) available with the original CrazyFlie 2.0, the small quadcopter used as baseline for building our prototype. Figure 8-B (called Big-S) shows the same CrazyFlie's motor coupled with a smaller blade. In Figure 8-C and -D, the Medium and Small motors are both paired with the smaller blade. All these motors and blades come from 
Thrust

\begin{tabular}{|c|c|c|}
\hline Motor Configuration & Measured & Interpolation \\
\hline Small & & - \\
\hline Medium & & - \\
\hline Big-S & $\boldsymbol{\nabla} \mathbf{v}$ & - \\
\hline Big-B & $\mathbf{\Delta}$ & \\
\hline
\end{tabular}

\begin{tabular}{|c|c|c|c|}
\hline MFDV & $0.1 \mathrm{~m} / \mathrm{s}$ & $0.2 \mathrm{~m} / \mathrm{s}$ & $0.3 \mathrm{~m} / \mathrm{s}$ \\
\hline Label & V0.1 & V0.2 & V0.3 \\
\hline
\end{tabular}
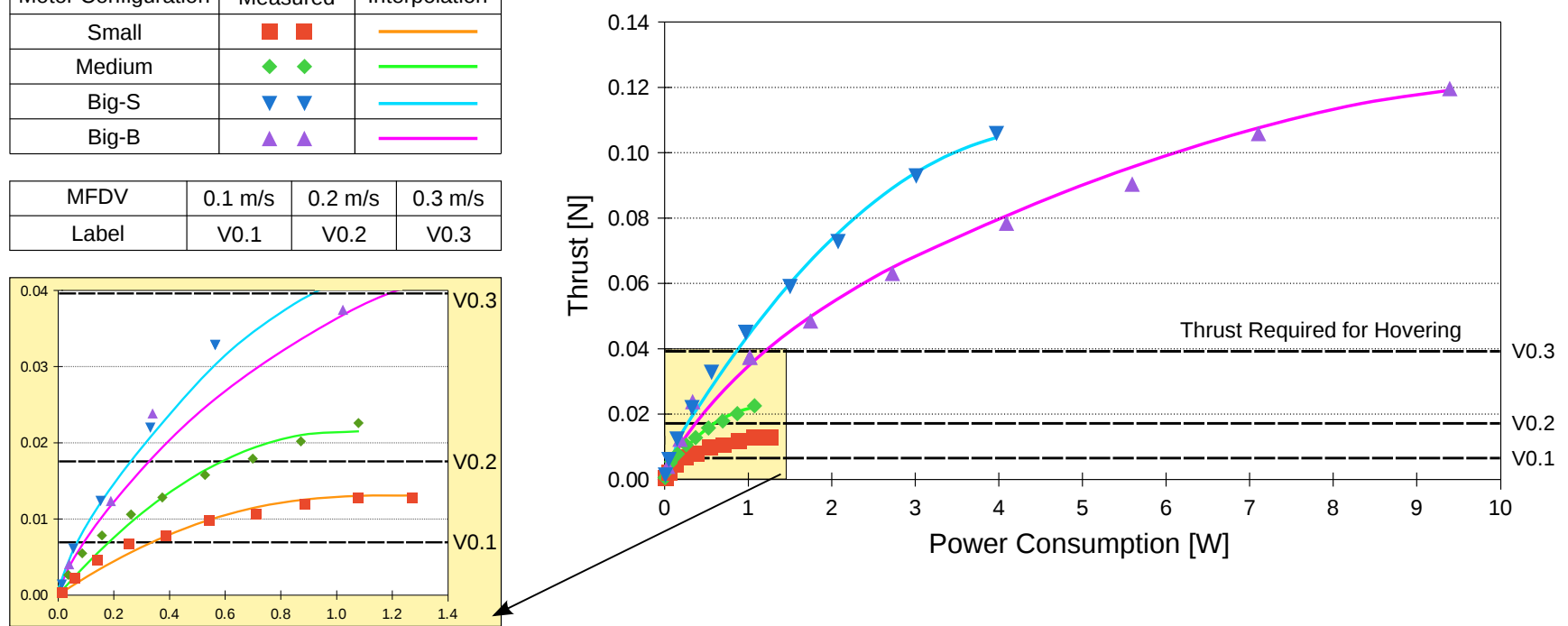

Fig. 9: Thrust vs. Power Consumption for 4 different motor configurations. With the dashed horizontal lines labeled "V0.1", "V0.2", "V0.3" is shown the thrust required to keep the blimp hovering, when the mean fall down velocity (MFDV) is respectively of $0.1,0.2$ and $0.3 \mathrm{~m} / \mathrm{s}$.

the "Do It Yourself" (DIY) RC community 6 and are commonly used for building custom UAVs.

In Figure 9, we evaluate the generated thrust and power consumption of the aforementioned motor configurations. All the motors considered are DC coreless motors, where the generated thrust can be controlled through the supply voltage in a pulse width modulation (PWM) fashion. The motors are evaluated in isolation w.r.t. the cyber-physical system, using a adjustable DC power supply. The y-axis of Figure 9 reports the measured thrust. This is calculated by measuring the speed of the air produced by each configuration using an anemometer. Thus, the thrust is computed as in Equation 7.

Thrust $=A_{\text {fan }} \times \delta_{\text {air }} \times$ Speed $_{\text {air }}^{2}$

Where $A_{\text {fan }}$ is the area of the airflow measured through the anemometer, $\delta_{\text {air }}$ is density of the air (i.e., $1.225 \mathrm{Kg} / \mathrm{m}^{3}$ ) and Speed $_{\text {air }}$ is the measured speed of the air. As expected, the generated thrust is proportional to the supply voltage and the configuration Big-B (Figure $8-\mathrm{A}$ ) is also the one which produces the greatest force.

On the $\mathrm{x}$-axis of Figure 9 the power consumption of each layout is reported. The Big-B configuration is the most power hungry due to the bigger blade and motor. In fact, such layout is able to produce up to $0.120 \mathrm{~N}$ of thrust, with a power consumption of $\sim 9 \mathrm{~W}$.

\footnotetext{
${ }^{6}$ http://www.microflight.com/Online-Catalog/Motors
}

The dashed horizontal lines, labeled "V0.1", "V0.2" and "V0.3", show the thrust required to keep the blimp hovering, when the blimp's mean fall down velocity (MFDV) is $0.1,0.2$ and $0.3 \mathrm{~m} / \mathrm{s}$, respectively. These values are computed as the average of the actual thrust values measured on four different prototypes and tuning the helium in order to obtain the desired MFDV velocities. This setup gives us the possibility to emulate the loss of helium that would occur after a long deflation time of the balloon (days and weeks).

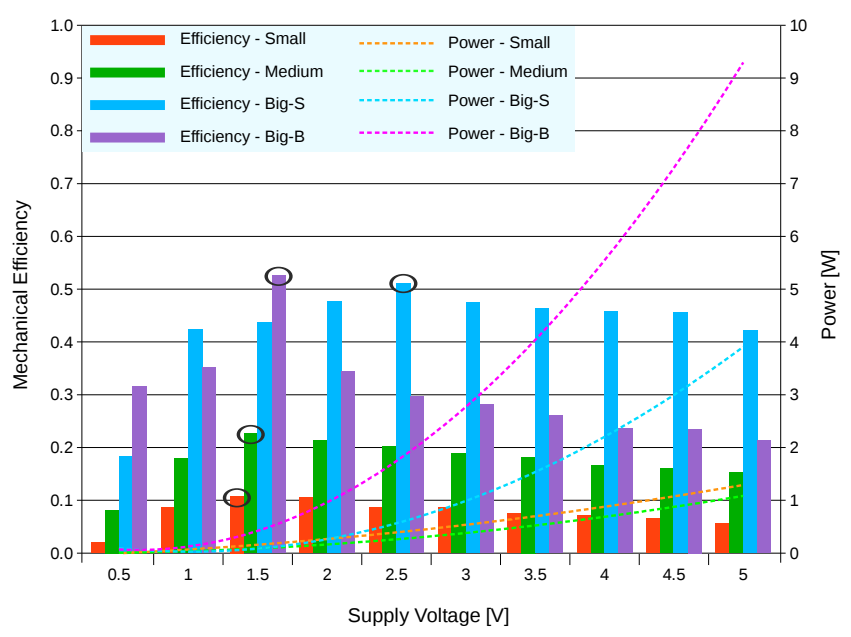

Fig. 10: Mechanical Efficiency/Power vs. Supply Voltage for the motor configurations evaluated. With the oval marker is highlighted the maximum efficiency point. 


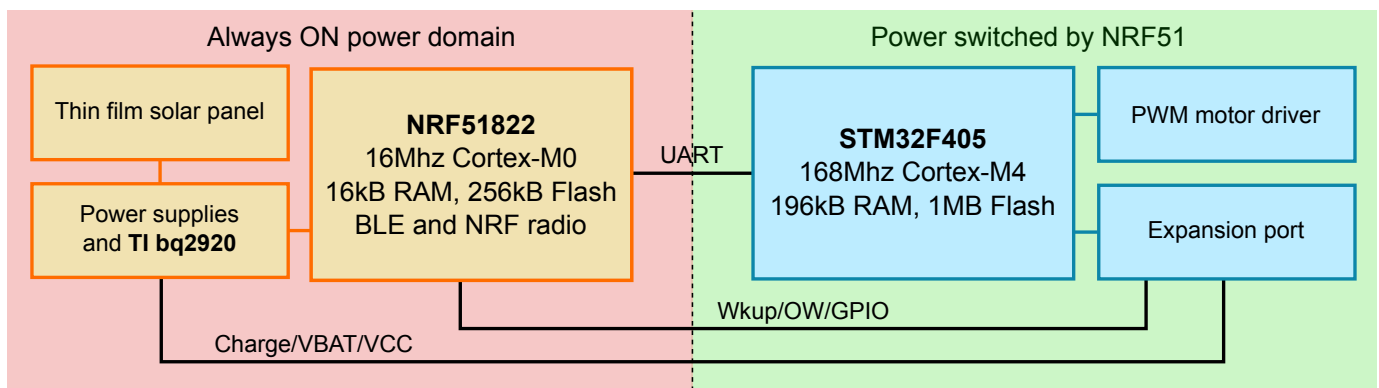

Fig. 11: Crazyflie2.0 electronics diagram.

The colored markers reported in Figure 9 show the actual measurements for the various motor configurations. As can be seen in Figure 9, only the Big-S and the $B i g-B$ configurations are able to provide enough thrust to counteract all the MFDV velocities considered. Even though the Medium configuration seems to be able to provide enough thrust to keep the system hovering with a fall down velocity of $0.2 \mathrm{~m} / \mathrm{s}$, this is not a feasible option since the required supply voltage $(3.8 \mathrm{~V})$ is beyond the blimp's battery maximum nominal voltage $(3.7 \mathrm{~V})$. Lastly, the Small configuration is able to provide enough push only to keep the system hovering when the fall down velocity is lower than $0.2 \mathrm{~m} / \mathrm{s}$.

The last part of our motors analysis aims to identify the best configuration in terms of mechanical efficiency (i.e., $\eta$ ). Such efficiency is computed as the ratio between the mechanical output power and the electrical input power, as shown in Equation 8.

$\eta=\frac{P_{\text {out }}}{P_{\text {in }}}=\frac{P_{\text {mec }}}{P_{\text {ele }}}=\frac{\text { Thrust } \times \text { Speed }_{\text {air }}}{\text { Power Consumption }}$

In Figure 10 we can see how the configurations Big-S and $B i g-B$ are the most efficient and both of them reach an efficiency of $\sim 0.5$. This is given by the fact that the evaluated efficiency is a property of the motor, thus the efficiency of the Big motor should be the same for both configurations. The small difference $(\sim 1 \%)$ shown in Figure 10, is given by: i) noise in the measurements and ii) coarse-grain discretization in the measurements (i.e., grown-step of $0.5 \mathrm{~V})$. Then, the maximum efficiency of the Small and Medium motors is significantly lower, respectively of $\sim 0.11$ and $\sim 0.23$. This is given by the fact that the motor's mechanical efficiency is proportional to the form factor, due to the constant power consumption overhead, for both electrical and physical reasons [14].

The power consumption, reported on the secondary y-axis of Figure 10, for the four configurations show that the peak performance is achieved with a power consumption of 340, 565, 87 and $94 \mathrm{~mW}$, respectively for Big-B, Big-S, Medium and Small. The Big-B configuration has the highest measurement noise due to the blade's dimension being significantly larger than the anemometer's fan $(45 \mathrm{~mm}$ w.r.t. $21 \mathrm{~mm}$ ). Since the power converter stage operates most efficiently at high voltages intensity, we select for our final prototype the Big-S motor configuration due to the required operational voltage range $(>3 V)$.

\subsection{Power Management in Software}

As was discussed in Section 3.2 and 3.3, we will study two different hovering mechanisms: continuously powering, and duty-cycling rotor. The former requires no special software mechanism since everything is kept on, while the latter requires additional control mechanisms to be discussed in this section.

In the original firmware the NRF51 is designated as the main processor. It controls the radio communication between the drone and the base station, and it controls the power supply to the sensors and the STM32 MCU. The developers system diagram [6] for the original drone can be seen in Figure 11. At the system start-up the NRF51 turns on the STM32 MCU, enabling its powerdomain. The STM32 firmware is based on a real time operating system. The operating system has a number of tasks that govern sensor reading, motor control, and communication between the two MCUs. The total

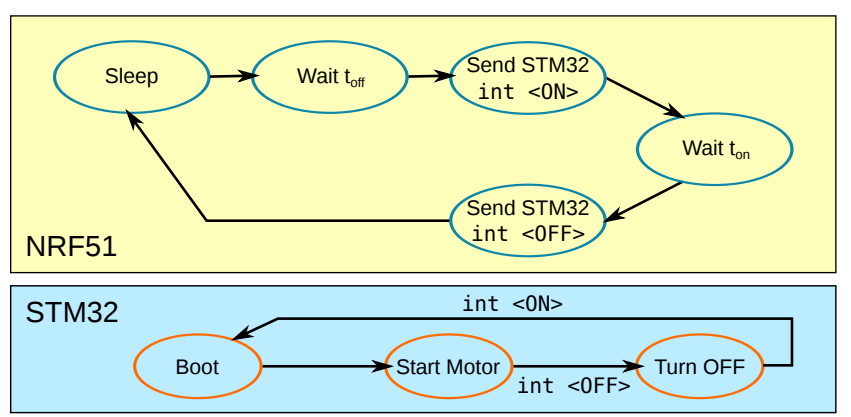

Fig. 12: State diagram of the NRF51 and STM32 MCU's. The 'int $\langle X\rangle$ ' labels indicate an interrupt for event $X$. 


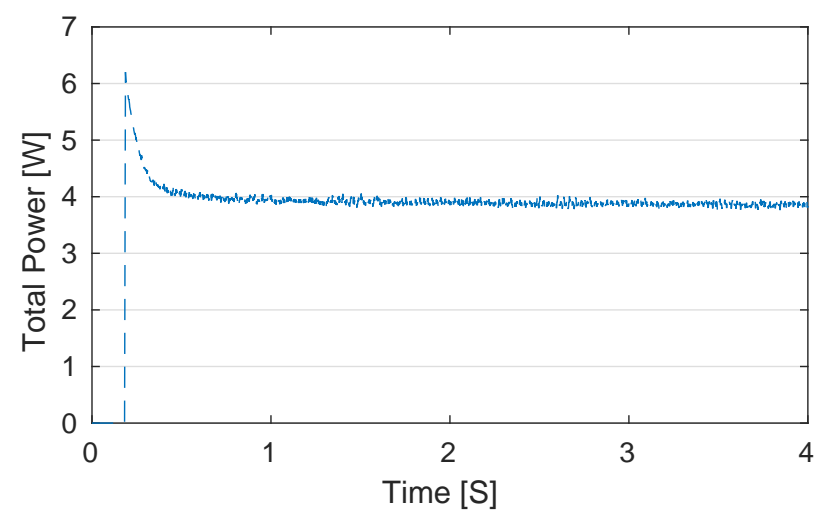

(a) Big-B power consumption.

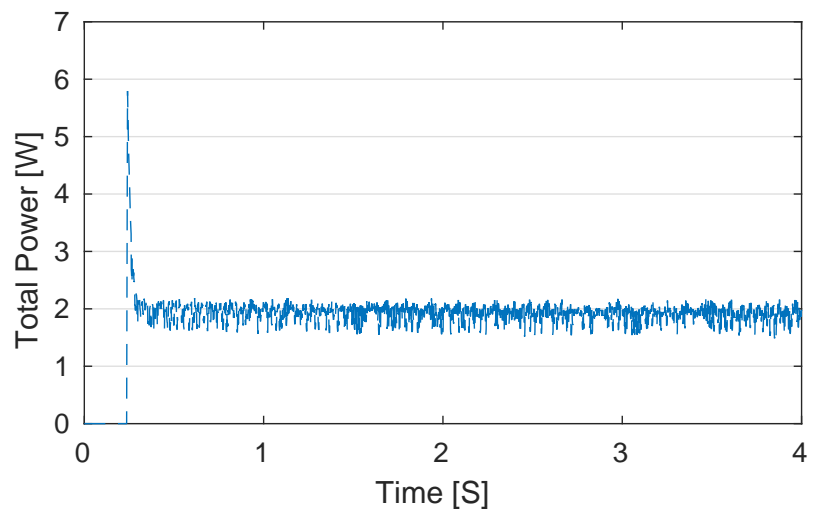

(b) Big-S power consumption.

Fig. 13: Power consumption of Big-B and Big-S configurations over 4 seconds @ 100\% thrust.

electronics power consumption, including the NRF51, the STM32 and sensors is $\sim 0.277 \mathrm{~W}$. To enable power cycling to conserve power during flight, our firmware version keeps only the functionality strictly required for our goal.

The proposed simplified, low-power firmware is presented in Figure 12. We kept the basic structure of the original firmware and we removed both the real-time operating system and the radio communication. The NRF51 and STM32 still govern the power distribution and the motor speed, respectively. The duty-cycling is enabled introducing in the NRF51 firmware a state machine that sets the on and off mode of the STM32. A timer in the same firmware is set to the desired duty cycle frequency and a master boot flag is set inside the interrupt, that is triggered by the timer. This boot flag controls the state machine. During the on phase it starts the STM32 and during the off phase it turns the STM32 off and drives the NRF51 to sleep mode to conserve power. The sleep portion of the code is critical to reducing the consumed power of the system. The power consumed during the off state is $\sim 5 \mu W$ and the power consumed during the on state is $\sim 4 W$ when the Big-B rotor is set to full intensity ( $\sim 2 W$ for Big-S). To differentiate the effect that the on-board electronics have on the blimp's lifetime, we will later evaluate an ideal blimp without on-board electronics.

As introduced in Section 3, under static power management (SPM) both duty-cycle and continuous mode operate with a static, predefined rotor intensity. The continuous mode can be enabled simply disabling the timer interrupt in the NRF51 firmware and boot the system directly to the STM32. When evaluating dynamic power management (DPM), we will focus on dynamically adjusting the duty-cycle. In software, this is done by updating the $t_{o f f}$ as the balloon deflates.

\section{System Evaluation}

In this section we present our prototype's experimental evaluation. We first introduce the initial measurements to characterize parameters like initialization overhead, rotor intensities/duty-cycle, and weight distribution. Then, we study the sustainability of both static power management (SPM) and dynamic power management (DPM), using both the Markov model presented in Section 4 as well as measurements using our prototype. For the sake of simplicity, our experiments will use a single weight distribution and assume constant harvesting conditions, unless otherwise stated.

\subsection{Initial Characterizations}

To get a better understanding of the basic parameters of a hovering blimp, and to be able to use them as input for our models, we have performed a set of initial tests to characterize our blimp implementation.

\section{Rotor Initialization Overhead}

All electric motors, including our blimp's coreless motor, have a power curve that peaks initially and then settles. This incurs an activation overhead that was discussed in Section 4.1. Figure 13a and 13b show the power consumption for the complete system using Big-B and Big-S configurations, respectively. The motors ran at $100 \%$ intensity for 4 seconds. To reduce they noise from the PWM regulation in the CrazyFlie 2.0, the power signals were passed through a low pass filter. In both cases, there is $\mathrm{a} \sim 6 \mathrm{~W}$ peak and after $\sim 0.3 \mathrm{~s}$ the system enters a steady state. The power consumption depends on the motor configuration and its intensity. In the case of Big-B, the average power consumption is $\sim 3.95 \mathrm{~W}$, while Big-S consumes $\sim 1.95 \mathrm{~W}$. 

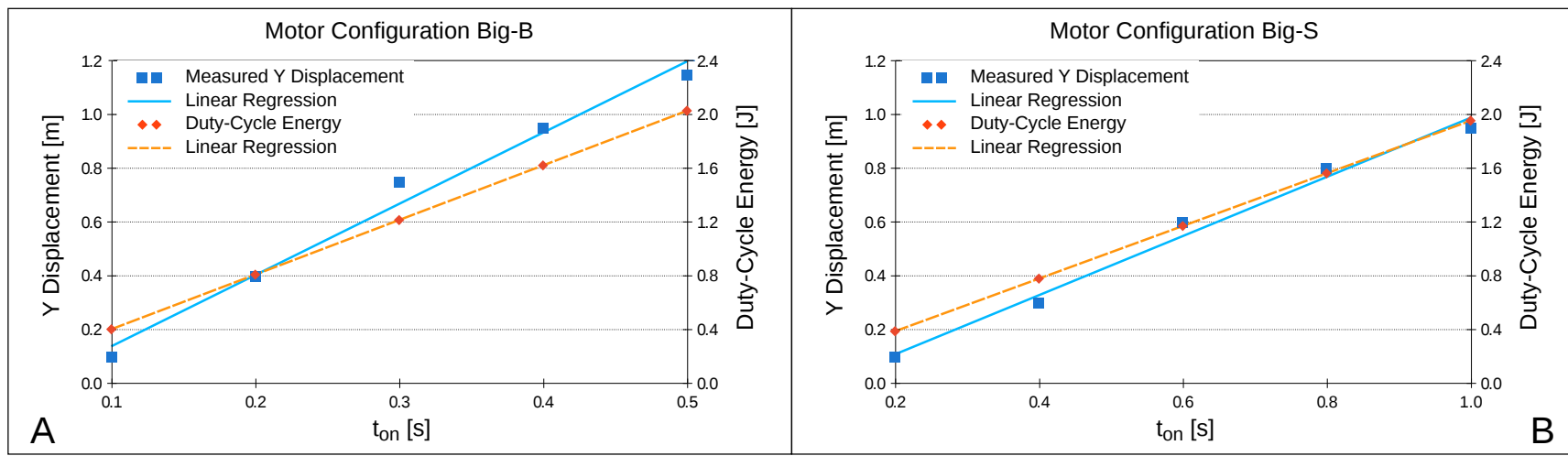

Fig. 14: Measured vertical displacement (Y) and system energy per period in duty-cycled blimp. A) motor configuration Big-B and B) the Big-S.

\section{Static Rotor Intensity and Duty-Cycle Selection}

Our baseline hovering technique uses constant thrust to compensate for gravity, thus maintaining the blimp at a constant altitude in a controlled environment. As was discussed in Section 3, the blimp requires relatively little rotor intensity to achieve this, thanks the lift provided by the helium. From our experiments, it was determined that only $9 \%$ rotor intensity was required for hovering (at MFDV of $0.1 \mathrm{~m} / \mathrm{s}$ ). This results in a total power consumption $P_{\text {cont }}=0.655 \mathrm{~W}$, for the system configured with Big-B. Instead, when the system uses the Big-S motor configuration, under the same assumptions, the measured total power consumption is $0.517 \mathrm{~W}$ (rotor intensity $10 \%$ ).

To determine the optimal duty cycle we conducted flight tests and measured the blimp's vertical displacement for different duty cycles and motor configurations. We have set our maximum height deviation, called $\Delta Y$, to be $\pm 25 \mathrm{~cm}$. Based on the collected data in Figure 14-A, we can see that one on period (i.e. $t_{o n}$ ) of $250 \mathrm{~ms}$ will cause the blimp to rise $50 \mathrm{~cm}$, with the Big-B motor configuration. This displacement takes longer than $250 \mathrm{~ms}$ due to the balloon's inertia. The $t_{\text {off }}$ needs to be long enough to allow the balloon to reach its maximum height and return to its initial position. This was experimentally determined to be $5 \mathrm{~s}$. With the selected duty-cycle of $t_{o n}=250 \mathrm{~ms}$ and $t_{o f f}=5 \mathrm{~s}$, the system has an average power consumption $P_{\text {duty }}=0.209 \mathrm{~W}$ (including the initialization overhead) and consumes $\sim 1.1 \mathrm{~J}$, as shown by the orange dashed line in Figure 14-A. Considering the Big-S motor configuration, a suitable duty-cycle configuration has been found in $t_{o n}=500 \mathrm{~ms}$ and $t_{o f f}=4.8 \mathrm{~s}$, as shown on Figure 14-B. This configuration has an average power consumption $P_{\text {duty }}=0.184 \mathrm{~W}$ and consumes $\sim 0.9 \mathrm{~J}$. Though our duty-cycle $t_{o n}$ is within the motor's current peak (for the
Big-B configuration), the average power consumption is still smaller than $P_{\text {cont }}$.

\section{Optimized Weight Distribution}

In order to analyze the weight distribution, we evaluate different batteries and solar panels sizes w.r.t. the available payload. As stated in Section 5, the blimp's maximum payload is $\sim 69 \mathrm{~g}$, but the actual weight budget we can spend for solar panel and battery is $40 \mathrm{~g}$, due to the $29 \mathrm{~g}$ used for the rotorcraft, connections and electronics. The evaluated weight combinations are reported on the $\mathrm{x}$-axis, with a growing step of $5 \mathrm{~g}$. The blimp's lifetime in duty-cycling mode was calculated for each weight distribution under two different environmental conditions: a constant insolation of $39 \mathrm{KLux}$ and 19.5 KLux.

In Figure 15-A we can see how, even with favorable lighting conditions, the blimp's lifetime first decreases from configuration $0 / 40$ to the $20 / 20$ before increasing from configuration $25 / 15$ to $40 / 0$. The peak, with an infinite lifetime, is reached with the $40 / 0$ configuration that represents the scenario where we use all the available payload for the solar panel. Although, this last case does not represent a feasible option in a real scenario due to the absence of any battery. The counterpart is represented by having only a $40 \mathrm{~g}$ battery without any solar panel and in this case the lifetime is 25 hours. In Figure 15-B, we can see how the limited insolation makes the solar panel unable to extend the blimp's lifetime. In fact, even using $35 \mathrm{~g}$ for the solar panel, we would obtain a lifetime of $\sim 7 \mathrm{~h}$.

From the previous payload distribution results, it was determined that our nano-blimp payload should be distributed in the following way: $8 g$ for the battery, and $31 \mathrm{~g}$ for the solar panel. This distribution coincides with commercially available products and ensures that the blimp can, under optimistic conditions, fly for possibly 


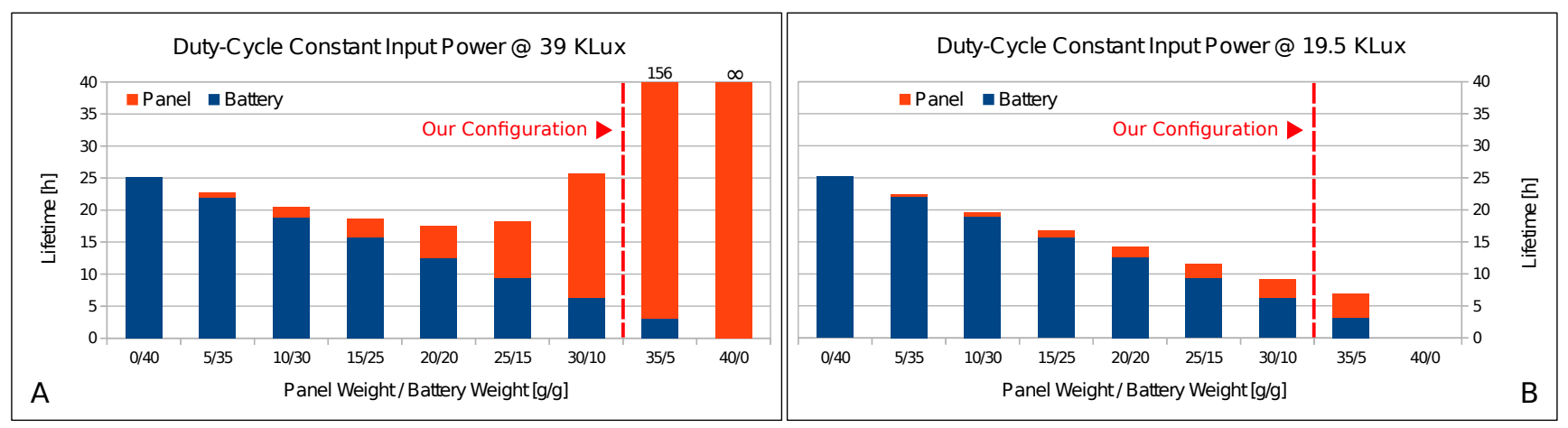

Fig. 15: Weight distribution evaluation for constant input power. A - @ 39 KLux and B - @ 19.5 KLux

over a hundred hours. At the same time, the blimp will have a minimum guaranteed flight time of several hours in pessimistic conditions.

\section{Battery Capacity}

The battery is, for the scope of our sustainability model, regarded as an ideal storage for energy. In reality the battery is not ideal, and we have observed, only a certain amount of energy can be drawn from a fully charged battery. Two rotor configurations were used to determine the amount of energy consumed by the load. The first configuration is when the rotor is running continuously, the other is duty cycled with a 0.25 second on and 5 second off time. The measurements show that the amount of energy that can be drawn depends on the rotor's configuration. We believe this difference to arise from the power peak necessary to turn on the rotors in the duty-cycling configuration. This requires a higher battery current and thus reduced the amount of available energy.

We will thus consider two ideal batteries in the model, the continuous configuration battery and the duty cycle configuration battery, and we use one depending on the rotor's configuration. The capacities of these two batteries were empirically obtained in the above mentioned measurements, and are 3156 Joules and 2767 Joules respectively. We assume in the model that the battery is always initially charged.

\section{Solar Panel Evaluation}

The selection of the solar panel is a key aspect for increasing the lifetime of the system. In fact, as previously shown in the weight distribution analysis, we have to select a solar panel that, for a given weight, can provide more energy than an equivalent battery. In order to understand how much power a solar panel can give us, in this section, we evaluate three different types of solar panels under the weight constraints imposed by our prototype. Given the blimp's payload and the base battery, the solar panel can weigh up to $32 \mathrm{~g}$. Using the Amorton product family ${ }^{7}$, we have analyzed the power densities for indoor and outdoor cells on a glass substrate as well as a thin film. After normalizing their maximum power points [32] by illuminance and by weight, the thin-film panel has a clear advantage since it can provide more power by virtue of offering a larger area for the available 32 grams and higher conversion efficiency. In Figure 16, we present the power that can be expected from the three panel types given different lighting conditions. The analyzed indoor and outdoor panels have been limited to illuminances ranging from $0-1.5 k L u x$ and $10-45 k L u x$, respectively. As shown in Figure 15, a blimp with an ideal balloon, duty-cycled rotor and a battery-only payload has at best 25 hours of system lifetime. As we discuss later in Section $6.3-\mathrm{dy}-$ namic model results - a blimp with a deflating balloon, duty-cycled rotor and a $32 g$ solar panel can reach the same lifetime when it harvests $\sim 210 \mathrm{~mW}$. Such power target is achieved by the thin film cell with $\sim 8.5 \mathrm{kLux}$, while the outdoor glass panel requires $\sim 43 k L u x$. While

\footnotetext{
7 https://panasonic.co.jp/es/pesam/en/products/
}

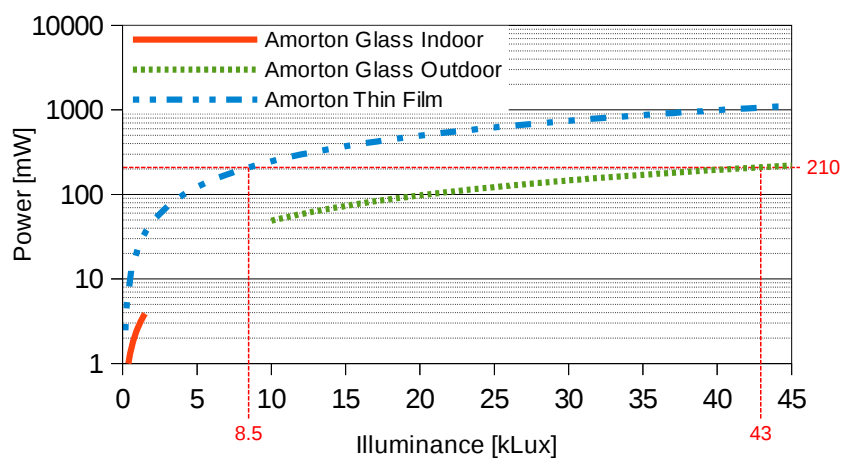

Fig. 16: Amorton solar panels comparison in their linear region of operation. 
$\sim 8.5 k L u x$ is higher than what is available in typical indoor scenarios [10], this is a small gap that will most likely be bridged by future solar cell technology (the required illuminance might also be already available in specific application scenarios like green-houses and industrial facilities).

\subsection{Static Power Management Evaluation}

The sustainability model, described in Section 4.1, was used to evaluate our prototype in order to estimate its lifetime. The model's estimates presented here are used to complement the experimental measurements. The results presented in this section are based on both Big$\mathrm{B}$ and Big-S motor configuration for comparison. For Dynamic Power Management, we will focus on Big-S, which has a lower power consumption.

\section{Static Model}

The blimp's parameters, including the battery capacity and the energy consumption, were based on the prototype's characterization. For the environmental conditions, two hypothetical scenarios were used: when the harvested energy is constant, and when the harvested energy follows a probability distribution. The consumed energy depends on the rotor's mode of operation as well. Experiments determining the rotor's consumption have been described in Section 6.1. Our sustainability model is a discrete time model whose parameters are reported in Table 2, both for Big-B and Big-S motor configurations.

\begin{tabular}{clccc}
\hline $\begin{array}{c}\text { Motor } \\
\text { Configuration }\end{array}$ & $\begin{array}{c}\text { Operation } \\
\text { Mode }\end{array}$ & $\begin{array}{c}\text { Period } \\
{[\mathrm{s}]}\end{array}$ & $\begin{array}{c}P_{\text {average }} \\
{[\mathrm{W}]}\end{array}$ & $\begin{array}{c}E_{\text {period }} \\
{[\mathrm{J}]}\end{array}$ \\
\hline \multirow{2}{*}{ Big-S } & Continuous & 5.30 & 0.517 & 2.740 \\
& Duty-Cycle & 5.30 & 0.184 & 0.975 \\
\hline \multirow{2}{*}{ Big-B } & Continuous & 5.25 & 0.655 & 3.439 \\
& Duty-Cycle & 5.25 & 0.209 & 1.097 \\
\hline
\end{tabular}

Table 2: SPM sustainability model parameters for both Big-B and Big-S motor configurations.

The harvested energy depends on the environmental conditions, and two hypothetical scenarios were used in the model. The first one was when the amount of energy harvested was the same for each time step. This constant scenario is fairly simple, and is an obvious choice for comparison with other results. The second environmental scenario used was when the harvested energy follows a logarithmic-normal ${ }^{8}$ distribution with a standard deviation of $\sigma=0.5$. It was chosen for the scenario as a first approximation of variable environment conditions [1, 33]. An example of a log-normal distribution used, with the mean value at $0.1 W$ and 0.5 standard deviation, is shown in Figure ??.

This second scenario considers volatile lighting conditions, where the energy harvested follows a probabilistic distribution. Before going into the results, we first need to comment on the definition of the lifetime, presented in (3). As stated in Section 4.1, the sustainability model provides us with the probability of the system being in a certain state after some time. Therefore, we need to define a threshold $\epsilon$, such that the system is defined not to work if the probability of the system being in the error state is larger than $\epsilon$. As (3) shows, the lifetime is a function of the $\epsilon$, so we estimated the lifetime using $\epsilon=10^{-4}$. A lifetime with $\epsilon=10^{-4}$ means that 999 blimps out of a 1000 are estimated to be working after this time. We shall call this $\epsilon$ choice the pessimistic case. The pessimistic case's lifetime estimation is influenced by periods that harvest low amounts of energy, even though these cases happen less often.

In Figure ??, the relative difference between the average and pessimistic lifetimes is shown. Note that the absolute values for these lifetimes will be discussed in the next section. What can be seen is that the pessimistic lifetime is estimated to be around 5\% shorter than the lifetime when energy is added constantly, and this difference increases as the lifetime rises.

\section{Static Model Results}

Using the prototype's specification explained in Section 6.1, we evaluate our proposed power management techniques for the nano-blimp. To this end, we use two configurations, one with continuous propulsion hovering and another with duty cycling. The parameters for both configuration were kept static, since we are evaluating SPM. For each configuration, we determined how different input power levels affect the blimp's lifetime.

Experiments with different input powers were set up for both configurations. The battery was initially charged for each experiment, and the blimp's lifetime was recorded. The lifetime is defined as the point at which the rotors stop producing enough lift to keep the blimp within the desired $\pm 25 \mathrm{~cm}$ altitude window. It should be noted that although the rotors continued to generate some lift after that, the battery was unable to supply the necessary power to maintain the blimp within the desired tolerance. This behavior was not

\footnotetext{
8 Logarithm of a normal distribution.
} 

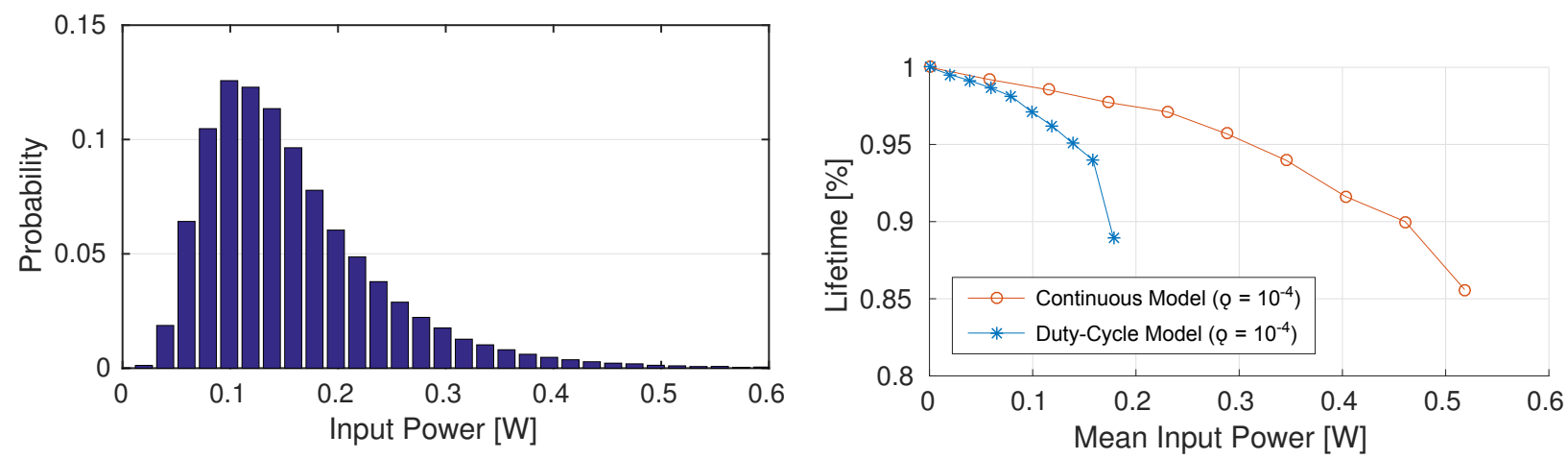

Fig. 17: Harvested power distribution in a scenario with variable input power, using Big-B configuration (left plot) and normalized lifetime results using the sustainability model for probabilistic harvesting (right plot).

considered correct w.r.t. the operative system and did not contribute to the lifetime.

Figure 18 shows the results of the experiments for both configurations using the Big-B motor configuration. The blimp's estimated lifetime refers to the scenario when the input energy is constant every time step. Note that the figure has the input power as the x-axis, and a period of 5.25 seconds is used to convert the power values to energy per period. The two lines in the plot represent the sustainability model results, described in the previous section. The marked points from each line indicate measurements made using the nano blimp prototype.

It should be noted that there is a vertical asymptote at $x=0.198 \mathrm{~W}$, which is the point at which selfsustainability is reached for duty-cycle mode. Due to the increased power requirements of continuous mode, this configuration's asymptote is located at $x=0.576 \mathrm{~W}$. The extended lifetimes of duty-cycle hovering clearly demonstrate the impact of the proposed power management. We can see how the measurements follow the model's predictions. As was mentioned in Section 3.4, the excess time is the system's lifetime without any input power $(x=0 \mathrm{~W})$. For the duty-cycle hovering configuration, the excess time is 3.78 hours, which is around $135 \%$ longer than the continuous hovering configuration.

In Figure 19, we present the lifetime model for the Static Power Management paired with the Big-S motor configuration and an ideal balloon with no deflation. We introduce the $P_{\text {elect }}$ parameter, used to represent the on-board microcontrollers introduced in Section 5.2. We consider two different power consumption scenarios: $P_{\text {elect }}=0 \mathrm{~W}$ is shown in Figure 19-A, and $P_{\text {elect }}=0.277 \mathrm{~W}$, shown in Figure 19-B. The former case represents the possibility either to turn the MCUs off or to enter in a deep-sleep mode where the MCUs' power consumption becomes negligible w.r.t. the rest of the system. The latter corner case (i.e., $P_{\text {elect }}=0.277 \mathrm{~W}$ ) represents all the computational resources always on for on-board processing.

Figure 19-A shows the most favorable conditions: ideal balloon and the MCUs' power consumption set to zero. In this case, the duty-cycle configuration is able to reach self-sustainability with an input power of $\sim 0.158 \mathrm{~W}$. The same is true for the continuous mode when the harvester is producing $\sim 0.240 \mathrm{~W}$. Figure

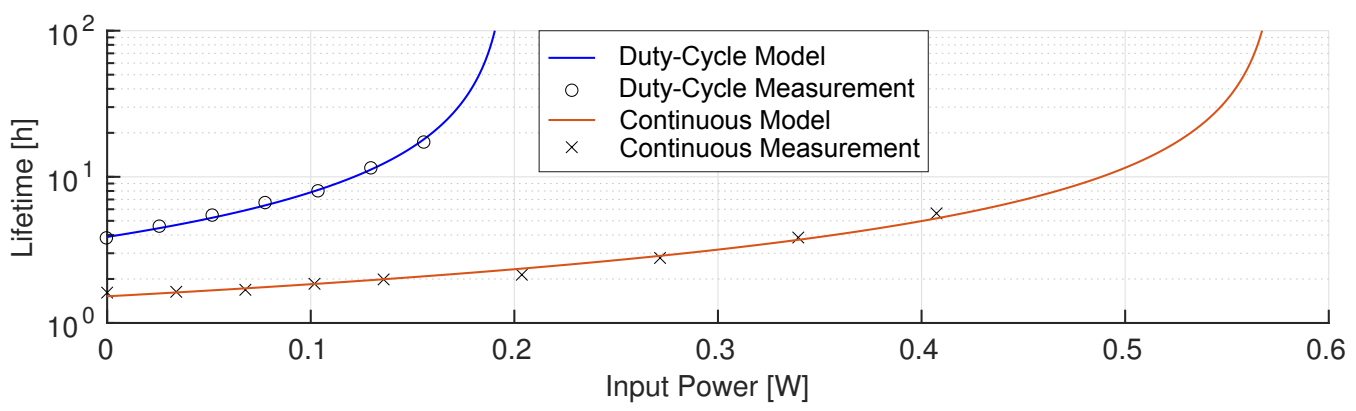

Fig. 18: Measured lifetimes as a function of constant input power for SPM with Big-B motor configuration. 


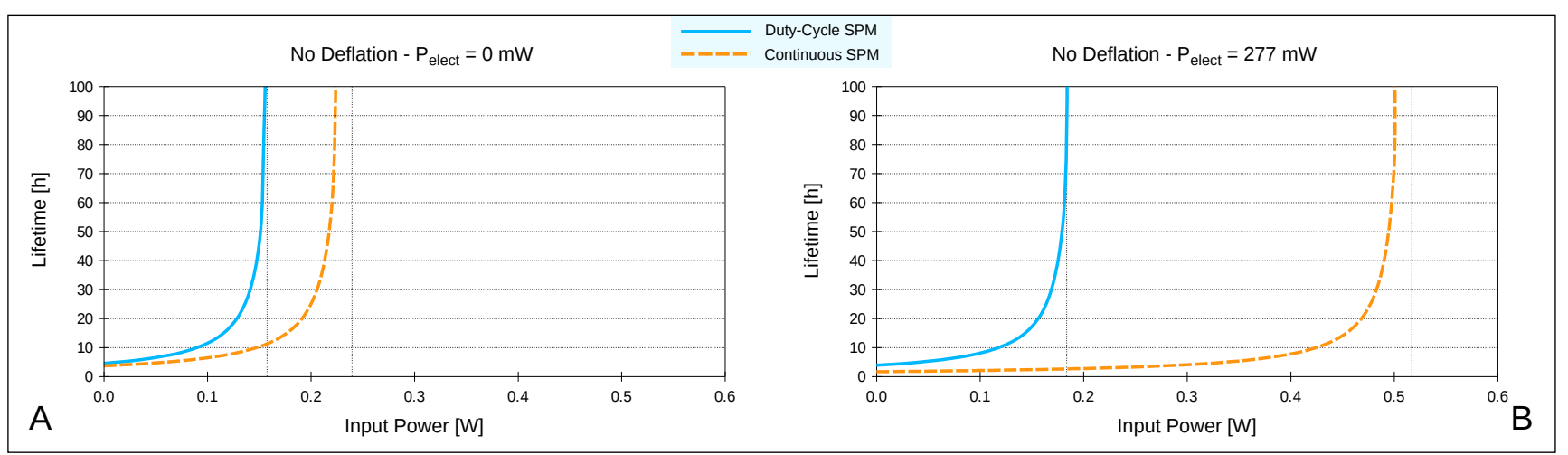

Fig. 19: Lifetime evaluation as a function of the input power without deflation. Static power management (SPM) with Big-S motor configuration. Evaluation with (B) and without (A) power consumption of the electronics (i.e., ( elect $)$.

19-B, shows the same condition shown in Figure 18, but with a different motor configuration. Under the assumption of no balloon deflation, the system reaches the self-sustainability with a constant input power of $\sim 0.184 \mathrm{~W}$ and $\sim 0.517 \mathrm{~W}$, for the duty-cycle and continuous configuration, respectively. Note that results in case of no deflation are independent from the specific power management technique (i.e., SPM or DPM), since the two power managements would behave exactly the same with an ideal balloon.

\subsection{Dynamic Power Management Evaluation}

In this section we evaluate dynamic power management (DPM) using experimental data without energy harvesting which is then extrapolated using our sustainability model to different harvesting conditions. In Section 6.2, static power management (SPM) was evaluated using both the duty-cycle configuration and the continuous mode. Also in this section we will evaluate the proposed DPM for both configurations, focusing on how its sustainability changes from the static to the dynamic case.

\section{Dynamic Model}

To understand how the duty-cycle period and intensity change with different motor/blade configurations and different lift conditions, we performed three different measurements for each mode. For each motor configuration, we calibrated the balloon to three different mean fall down velocities (MFDVs). These represent different levels of deflation, as was first presented in Section 3.3. The duty-cycle parameters are obtained through the experimental analysis of the prototypes we built. As introduced in Section 6.1, the $t_{\text {on }}$ is defined as the time required to go up $50 \mathrm{~cm}$, and was measured after calibrating the balloon's lift for a given MFDV. The $t_{\text {off }}$ period is then adjusted, through multiple experiments, until the desired hovering behavior is obtained. For the continuous mode we experimentally identified the required motor intensity for keep it hovering under different inflation of the balloon. Afterwards, the lifetimes for each setup were measured using an initially charged battery and no energy harvesting $\left(P_{i n}=0\right)$.

The duty-cycle and continuous configurations are, respectively, reported in Table 3 and Table 4 . All the motors (described in Section 5.15 .1 ) have been characterized under each of these configurations, simulating different deflations of the balloon. Such configurations are experimentally identified in order to keep the blimp hovering in presence of three different MFDVs (i.e., 0.1, 0.2 and $0.3 \mathrm{~m} / \mathrm{s}$ ). In order to obtain the desired MFDV, we regulated the amount of helium in the balloon for each case. The parameters in Table 3 show the voltage intensity during the $t_{\text {on }}$ and the duration of the $t_{\text {on }}$ and $t_{\text {off }}$ period. Instead, the parameters in Table 4 show the software intensity and the consequent voltage intensity required by each motor. In the last column of both tables we also report the measured lifetimes for each configuration under the assumption of no input power coming from the solar harvester (i.e., $P_{i n}=0$ ).

As already discussed in Section 5.1, the Small and Medium configurations are able to keep the vehicle hovering only when the MFDV is $0.1 \mathrm{~m} / \mathrm{s}$ or lower. Then, considering the Big-S and Big-B configurations, it is clear that a higher MFDV requires a higher average thrust. For the duty-cycle mode, shown in Table 3, the average thrust is adjusted by both increasing the $t_{o n}$ and decreasing the $t_{o f f}$. Due to its ability to tolerate higher MFDVs and maximize the blimp's lifetime, we select the Big-S motor type for our self-sustainability study. 
Its different duty-cycle and continuous settings are the discrete points which are used to build the fine-grained (i.e., continuous) lifetime models discussed in the next section.

\begin{tabular}{cccccc}
\hline $\begin{array}{c}\text { Motor } \\
\text { Configu- } \\
\text { ration }\end{array}$ & $\begin{array}{l}\text { MFDV } \\
{[\mathrm{m} / \mathrm{s}]}\end{array}$ & $\begin{array}{c}t_{\text {on }} \\
{[\mathrm{ms}]}\end{array}$ & $\begin{array}{c}t_{\text {off }} \\
{[\mathrm{ms}]}\end{array}$ & $\begin{array}{c}\text { Volt- } \\
\text { age } \\
{[\mathrm{V}]}\end{array}$ & $\begin{array}{c}\text { Measured } \\
\text { Lifetime }[\mathrm{h}: \mathrm{m}: \mathrm{s}] \\
\left(P_{\text {in }}=0\right)\end{array}$ \\
\hline \multirow{3}{*}{ Small } & 0.1 & 3000 & 3400 & & $02: 45: 58$ \\
& 0.2 & - & - & 3.7 & - \\
\hline \multirow{3}{*}{ Medium } & 0.3 & - & - & & - \\
& 0.1 & 1900 & 3500 & & $03: 06: 25$ \\
& 0.2 & - & - & 3.7 & - \\
\hline & 0.1 & 500 & 4800 & & $04: 23: 18$ \\
Big-S & 0.2 & 700 & 2240 & 3.6 & $01: 49: 05$ \\
& 0.3 & 1600 & 1150 & & $00: 44: 43$ \\
\hline & 0.1 & 250 & 5000 & & $04: 05: 11$ \\
Big-B & 0.2 & 350 & 2220 & 3.4 & $01: 16: 05$ \\
& 0.3 & 520 & 1400 & & $00: 37: 22$ \\
\hline
\end{tabular}

Table 3: Dynamic power management configurations for different motors and MFDVs, running in duty-cycle mode. The measured lifetime is with battery only $\left(P_{i n}=\right.$ $0)$.

\begin{tabular}{ccccc}
\hline $\begin{array}{c}\text { Motor } \\
\text { Configu- } \\
\text { ration }\end{array}$ & $\begin{array}{c}\text { MFDV } \\
{[\mathrm{m} / \mathrm{s}]}\end{array}$ & $\begin{array}{c}\text { SW In- } \\
\text { tensity } \\
{[\%]}\end{array}$ & $\begin{array}{c}\text { Volt- } \\
\text { age } \\
{[\mathrm{V}]}\end{array}$ & $\begin{array}{c}\text { Measured } \\
\text { Lifetime [h:m:s] } \\
\left(P_{\text {in }}=0\right)\end{array}$ \\
\hline \multirow{3}{*}{ Small } & 0.1 & 48 & 1.98 & $01: 32: 41$ \\
& 0.2 & - & - & - \\
\hline \multirow{3}{*}{ Medium } & 0.3 & - & - & - \\
& 0.1 & 39 & 1.79 & $01: 31: 55$ \\
& 0.2 & - & - & - \\
Big-S & 0.3 & - & - & - \\
& 0.2 & 10 & 1.11 & $01: 40: 41$ \\
Big-B & 0.3 & 62 & 2.79 & $01: 10: 15$ \\
& 0.1 & 9 & 0.62 & $01: 39: 45$ \\
\hline
\end{tabular}

Table 4: Dynamic power management configurations for different motors and MFDVs, running in continuous mode. The measured lifetime is with battery only $\left(P_{i n}=\right.$ $0)$.

\section{Dynamic Model Results}

The dynamic power management (DPM) lifetime model is fairly similar to the SPM one presented in Section 6.2. The ideal battery and harvesting is kept the same. What is different in the DPM case is the duty-cycle period, the motor intensity in continuous mode and the energy consumption, as shown in Table 3 and Table 4 . In our fine-grained model, we shall use a single period, meaning that once per period energy is removed from the battery. The energy consumed per period is extrapolated from the rotor's measured voltage, $t_{o n}$ and $t_{\text {off }}$ values (for the continuous mode we can consider $t_{o f f}=0$ every period). What we can observe is that the energy consumed per period, as well as $t_{o n} /\left(t_{o n}+t_{o f f}\right)$, increases quadratically with the MFDV.

Figure 20 shows the blimp's simulated lifetime for the DPM in case of deflation. Similarly to the SPM evaluation in Section 6.2, we consider a power consumption for the microcontrollers $\left(P_{\text {elect }}\right)$ that spans from an ideal minimum of $0 \mathrm{~W}$, shown in Figure 20-A, to a maximum of $0.277 \mathrm{~W}$, shown in Figure 20-B. On the y-axis we report the expected system's lifetime as a function of the constant input power coming from the solar harvesting (x-axis).

Figure 20 depicts the system lifetime in case of deflation, using the best performing motor configuration: Big-S. It shows the system's lifetime when it is able to dynamically adjust its configuration according to the proposed DPM. In the duty-cycle configuration $t_{\text {on }}$ and $t_{\text {off }}$ periods are adjusted every $\sim 5 \mathrm{~s}$, increasing the power consumption with the same fine-grained resolution. Similarly, in continuous mode the motor intensity is increased with the same granularity. In both Figure 20-A and -B is possible to see that by duty-cycling the system, we obtain a longer lifetime w.r.t. the continuous mode. Obviously, this difference increases in Figure 20-B due to the higher $P_{\text {elect }}$.

The two plots also show three areas representing the MFDV the system would have after some time due to the deflation of the balloon. From both Figure 20-A and -B is noticeable how the two configurations converge for high input powers. The increased input power extends the blimp's lifetime, making deflation more relevant since it causes higher MFDVs. As the MFDV increases, the duty-cycle mode needs to increase the $t_{\text {on }}$ and to reduce the $t_{\text {off }}$. Similarly, the continuous mode needs to increase the rotor intensity. The limit to this convergence is represented by the lifetime's upper bound $(\sim 410 h)$, where the two modes will behave exactly the same. After that time, the balloon would be so deflated that it is impossible keep it hovering even if the motor is always on at the maximum intensity. 


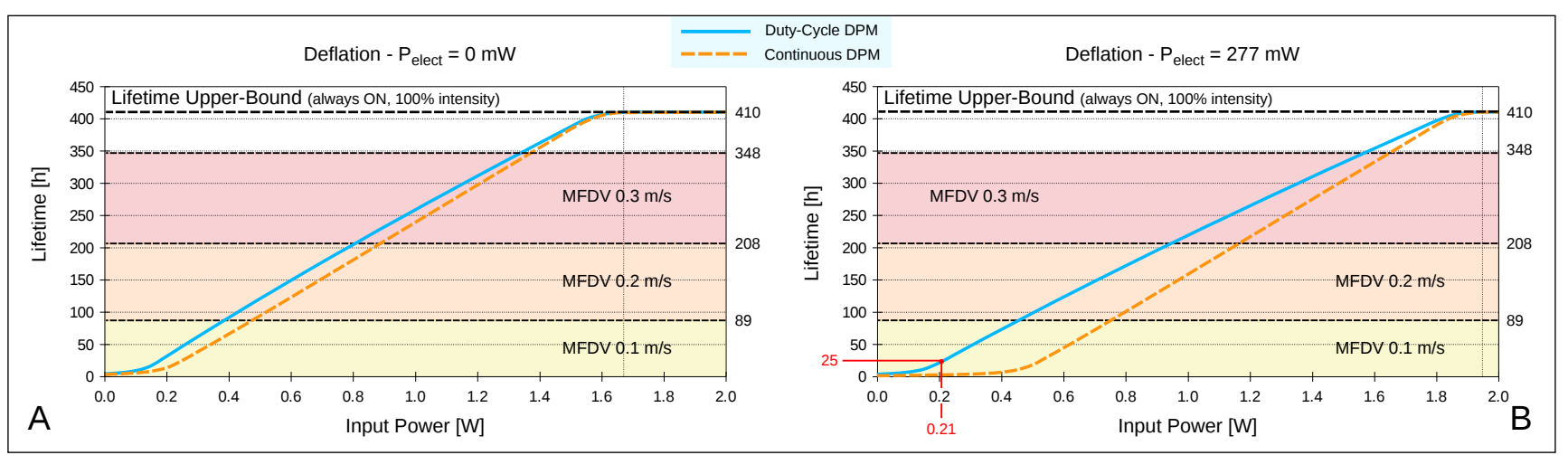

Fig. 20: Lifetime evaluation as a function of the input power with deflation. Dynamic power management (DPM) with Big-S motor configuration. Evaluation with (B) and without (A) power consumption of the electronics (i.e., $\left.P_{\text {elect }}\right)$.

When comparing the lifetime of SPM w.r.t. DPM in presence of deflation, it is clear that after some time, SPM would not be able to hover anymore. Even in an optimistic case, SPM would not last more than $89 h$ which is when the MFDV increases from 0.1 to $0.2 \mathrm{~m} / \mathrm{s}$. At that time, the system with SPM is unable to dynamically adjust its motor configuration. It would not be able to compensate for the loss of helium, meaning the blimp would fall to the ground and the mission would terminate, even if some energy would still be available in the battery.

Thus, the dynamic power management gives us a lifetime extension at least from $\sim 89 h$ to $\sim 410 h$, w.r.t. the static power management. The price for such an extension in the lifetime is a constant input power of $\sim 1.67 \mathrm{~W}$ and $\sim 1.95 \mathrm{~W}$ coming from the solar panel, respectively in case of $P_{\text {elect }}=0 \mathrm{~W}$ and $P_{\text {elect }}=0.277 \mathrm{~W}$. At the same time this result indicates that a larger input power would not extend the system lifetime, placing a threshold on the useful input power from the harvester.

In Figure 20-B we also highlight (in red) the input power required to achieve a lifetime of $\sim 25$ hours, which is the maximum duration of an ideal, duty-cycled, battery-only blimp. Whenever a solar panel harvests more than $210 \mathrm{~mW}$, we can obtain a lifetime longer than that of a battery-only blimp.

\section{Conclusions}

As nano-UAVs have become more ubiquitous in recent years, their short lifetimes have been a limiting factor in many application domains. We have presented a nano-blimp platform that leverages energy harvesting and power management techniques to achieve significantly extended lifetimes. Due to power converters, duty-cycling the on-board high-intensity rotor is more energy-efficient than low-intensity, continuous operation. However, to really hover for long periods it is essential to dynamically adjust the rotor configuration to the blimp's deflation. Dynamic parameter reconfiguration allows the blimp to reach lifetimes of up to $\sim 400$ hours under optimistic harvesting conditions. This constitutes an improvement of over $4 \times$ compared to the same system without the proposed dynamic parameter reconfiguration.

Furthermore, using a sustainability model, we have analyzed how different power management techniques can extend a nano-blimp's lifetime under different harvesting and load conditions. Extensive experimental results have demonstrated the validity of our model and power management strategies.

This work lays the foundation for an energy autonomous nano-blimp. The relaxed energy constraint leaves a margin to implement more sophisticated cognitive skills (e.g., autonomous navigation, path planning, etc.). As part of our future work we plan to extend the system's capability to adapt to changing environmental conditions (e.g., temperature, humidity and pressure).

Acknowledgements The authors thank Arnaldo Palossi, Lukas Sigrist and Antonio Pullini for their support.

\section{References}

1. Comparison of energy intake prediction algorithms for systems powered by photovoltaic harvesters. Microelectronics Journal 41(11), 766 - 777 (2010)

2. P1110b - $915 \mathrm{mhz}$ rf powerharvester receiver (2016)

3. Achtelik, M.C., Stumpf, J., Gurdan, D., Doth, K.M.: Design of a flexible high performance quadcopter platform breaking the mav endurance record with laser power beaming. In: Intelligent robots and systems (iros), 2011 ieee/rsj international conference on, pp. 5166-5172. IEEE (2011) 
4. Benini, L., Bogliolo, A., De Micheli, G.: A survey of design techniques for system-level dynamic power management. IEEE transactions on very large scale integration (VLSI) systems 8(3), 299-316 (2000)

5. Bhatti, N.A., Alizai, M.H., Syed, A.A., Mottola, L.: Energy harvesting and wireless transfer in sensor network applications: Concepts and experiences. ACM Transactions on Sensor Networks (TOSN) 12(3), 24 (2016)

6. Bitcraze: System diagram (2016)

7. Briod, A., Zufferey, J.C., Floreano, D.: Optic-flow based control of a $46 \mathrm{~g}$ quadrotor. In: Workshop on Vision-based Closed-Loop Control and Navigation of Micro Helicopters in GPS-denied Environments, IROS 2013, EPFL-CONF189879 (2013)

8. Burri, M., Gasser, L., Käch, M., Krebs, M., Laube, S., Ledergerber, A., Meier, D., Michaud, R., Mosimann, L., Müri, L., Ruch, C., Schaffner, A., Vuilliomenet, N., Weichart, J., Rudin, K., Leutenegger, S., Alonso-Mora, J., Siegwart, R., Beardsley, P.: Design and control of a spherical omnidirectional blimp. In: 2013 IEEE/RSJ International Conference on Intelligent Robots and Systems, pp. 1873-1879 (2013). DOI 10.1109/IROS.2013.6696604

9. Cocchioni, F., Mancini, A., Longhi, S.: Autonomous navigation, landing and recharge of a quadrotor using artificial vision. In: Unmanned Aircraft Systems (ICUAS), 2014 International Conference on, pp. 418-429. IEEE (2014)

10. EnOcean: Application note 201: Indoor lighting conditions - placement of solar powered sensor units (2015)

11. Falanga, D., Mueggler, E., Faessler, M., Scaramuzza, D.: Aggressive quadrotor flight through narrow gaps with onboard sensing and computing. arXiv preprint arXiv:1612.00291 (2016)

12. Ghosh, K., Guha, A., Duttagupta, S.P.: Power generation on a solar photovoltaic modules integrated lighterthan-air platform at a low altitude. arXiv preprint arXiv:1610.07598 (2016)

13. Gomez, A., Sigrist, L., Magno, M., Benini, L., Thiele, L.: Dynamic energy burst scaling for transiently powered systems. In: Proc. DATE Conf., pp. 349-354. EDA Consortium (2016)

14. Harrington, A.M., Kroninger, C.: Characterization of small dc brushed and brushless motors. Tech. rep., ARMY RESEARCH LAB ABERDEEN PROVING GROUND MD VEHICLE TECHNOLOGY DIRECTORATE (2013)

15. Kim, J., Song, B.D., Morrison, J.R.: On the scheduling of systems of uavs and fuel service stations for long-term mission fulfillment. Journal of Intelligent \& Robotic Systems pp. 1-13 (2013)

16. Knaupp, W., Mundschau, E.: Photovoltaic-hydrogen energy systems for stratospheric platforms. In: 3rd World Conference onPhotovoltaic Energy Conversion, 2003. Proceedings of, vol. 3, pp. 2143-2147 Vol.3 (2003)

17. Kurs, A., Karalis, A., Moffatt, R., Joannopoulos, J.D., Fisher, P., Soljačić, M.: Wireless power transfer via strongly coupled magnetic resonances. science $\mathbf{3 1 7}(5834)$, 83-86 (2007)

18. Leutenegger, S.: Unmanned solar airplanes: Design and algorithms for efficient and robust autonomous operation. Ph.D. thesis, ETH-Zürich (2014)

19. Leutenegger, S., Jabas, M., Siegwart, R.Y.: Solar airplane conceptual design and performance estimation. In: In Proceedings of the 3rd International Symposium on Unmanned Aerial Vehicles (2010)

20. Li, J., Lv, M., Sun, K.: Optimum area of solar array for stratospheric solar-powered airship. Proceedings of the Institution of Mechanical Engineers, Part G: Journal of Aerospace Engineering p. 0954410016670420 (2016)
21. Li, P., Garratt, M., Lambert, A.: Monocular snapshotbased sensing and control of hover, takeoff, and landing for a low-cost quadrotor. Journal of Field Robotics 32(7) (2015). DOI 10.1002/rob.21574

22. Liang, H., Zhu, M., Guo, X., Zheng, Z.: Conceptual design optimization of high altitude airship in concurrent subspace optimization. In: 50th AIAA aerospace sciences meeting including the new horizons forum and aerospace exposition, p. 1180 (2012)

23. Lozano, R.: Unmanned aerial vehicles: Embedded control. John Wiley \& Sons (2013)

24. Noll, T.E., Brown, J.M., Perez-Davis, M.E., Ishmael, S.D., Tiffany, G.C., Gaier, M.: Investigation of the helios prototype aircraft mishap volume i mishap report. Downloaded on 9, 2004 (2004)

25. Nugent, T.J., Kare, J.T.: Laser power for uavs. Laser Motive White Paper-Power Beaming for UAVs, NWEN (2010)

26. Nugent, T.J., Kare, J.T.: Laser power beaming for defense and security applications. In: Unmanned Systems Technology XIII, vol. 8045, p. 804514. International Society for Optics and Photonics (2011)

27. Oettershagen, P., Melzer, A., Mantel, T., Rudin, K., Lotz, R., Siebenmann, D., Leutenegger, S., Alexis, K., Siegwart, R.: A solar-powered hand-launchable uav for low-altitude multi-day continuous flight. In: Robotics and Automation (ICRA), 2015 IEEE International Conference on, pp. 39863993. IEEE (2015)

28. Palossi, D., Furci, M., Naldi, R., Marongiu, A., Marconi, L., Benini, L.: An energy-efficient parallel algorithm for real-time near-optimal uav path planning. In: Proceedings of the ACM International Conference on Computing Frontiers, CF '16, pp. 392-397. ACM, New York, NY, USA (2016). DOI $10.1145 / 2903150.2911712$. URL http://doi.acm.org/10.1145/2903150.2911712

29. Palossi, D., Gomez, A., Draskovic, S., Keller, K., Benini, L., Thiele, L.: Self-sustainability in nano unmanned aerial vehicles: A blimp case study. In: Proceedings of the Computing Frontiers Conference, pp. 79-88. ACM (2017)

30. Palossi, D., Marongiu, A., Benini, L.: Ultra low-power visual odometry for nano-scale unmanned aerial vehicles. In: 2017 Design, Automation \& Test in Europe Conference \& Exhibition (DATE), pp. 1647-1650. IEEE (2017)

31. Palossi, D., Singh, J., Magno, M., Benini, L.: Target following on nano-scale unmanned aerial vehicles. In: Advances in Sensors and Interfaces (IWASI), 2017 7th IEEE International Workshop on, pp. 170-175. IEEE (2017)

32. Panasonic: Amorphous silicon solar cells (2014). URL https://panasonic.co.jp/es/pesam/en/products/pdf/ Catalog_Amorton_ENG.pdf

33. Park, J., Liang, W., Choi, J., El-Keib, A., Shahidehpour, M., Billinton, R.: A probabilistic reliability evaluation of a power system including solar/photovoltaic cell generator. In: Power \& Energy Society General Meeting, 2009. PES'09. IEEE, pp. 1-6. IEEE (2009)

34. Shaheed, M.H., Abidali, A., Ahmed, J., Ahmed, S., Burba, I., Fani, P.J., Kwofie, G., Wojewoda, K., Munjiza, A.: Flying by the sun only: The solarcopter prototype. Aerospace Science and Technology 45, 209-214 (2015)

35. Siciliano, B., Khatib, O.: Springer handbook of robotics. Springer (2016)

36. Toksoz, T., Redding, J., Michini, M., Michini, B., How, J.P., Vavrina, M., Vian, J.: Automated battery swap and recharge to enable persistent uav missions. In: AIAA Infotech@ Aerospace Conference (2011)

37. Verling, S., Weibel, B., Boosfeld, M., Alexis, K., Burri, M., Siegwart, R.: Full attitude control of a vtol tailsitter 
uav. In: 2016 IEEE International Conference on Robotics and Automation (ICRA), pp. 3006-3012 (2016). DOI 10.1109/ICRA.2016.7487466

38. Weider, A., Levy, H., Regev, I., Ankri, L., Goldenberg, T., Ehrlich, Y., Vladimirsky, A., Yosef, Z., Cohen, M.: Sunsailor: solar powered uav. Technion IIT, Haifa, Israel (2006)

39. Wood, R., Finio, B., Karpelson, M., Ma, K., PérezArancibia, N., Sreetharan, P., Tanaka, H., Whitney, J.: Progress on 'pico' air vehicles. Int. J. Rob. Res. 31(11) (2012). DOI 10.1177/0278364912455073

40. Zhang, X., Xian, B., Zhao, B., Zhang, Y.: Autonomous flight control of a nano quadrotor helicopter in a gpsdenied environment using on-board vision. IEEE Transactions on Industrial Electronics 62(10), 6392-6403 (2015)

41. van der Zwaan, S., Bernardino, A., Santos-Victor, J.: Vision based station keeping and docking for an aerial blimp. In: Intelligent Robots and Systems, 2000.(IROS 2000). Proceedings. 2000 IEEE/RSJ International Conference on, vol. 1, pp. 614-619. IEEE (2000)

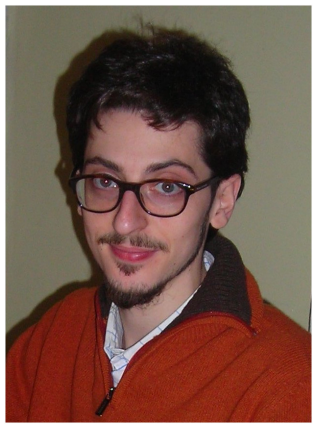

Daniele Palossi is a Ph.D. student at the Dept. of Information Technology and Electrical Engineering at the Swiss Federal Institute of Technology in Zürich (ETH Zürich). He received his B.S. and M.S. in Computer Science Engineering from the University of Bologna, Italy. In 2012 he spent 6 months as a research intern at ST Microelectronics, Agrate Brianza, Milano, working on 3D computer vision algorithms for the STM STHORM project. In 2013 he won a one-year research grant at the University of Bologna, with a focus on design methodologies for high-performance embedded systems. He is currently working on energy-efficient algorithms for autonomous vehicles and advanced driver assistance systems.

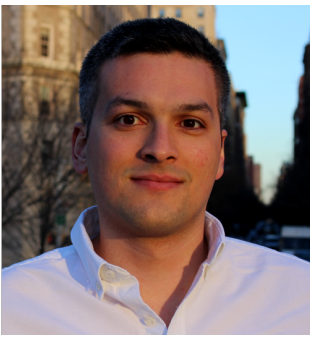

Andres Gomez is a currently a Ph.D. student at ETH Zürich. He obtained his MSc degree in Embedded Systems Design in 2014 from the ALaRI institute at the Università della Svizzera Italiana, Switzerland. Previously, he obtained a dual degree in electronics engineering and systems engineering in 2011 from the Universidad de Los Andes, Colombia. His main research interests are low power system design and transient computing systems, in particular $\mathrm{HW} / \mathrm{SW}$ codesign for energy-efficient system operation.

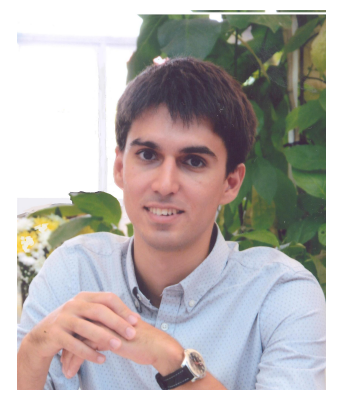

Stefan Draskovic received the MSc degree in electrical engineering from ETH Zürich, and is currently working toward a $\mathrm{Ph} . \mathrm{D}$. degree at the same university. His research interests cover multiprocessor system design and performance analysis.

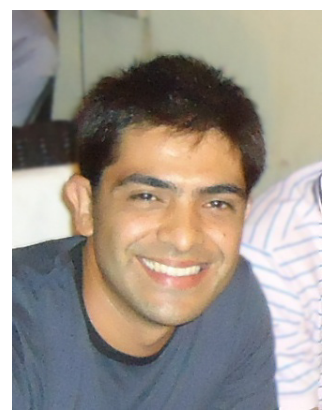

Andrea Marongiu received the MSc degree in electronic engineering from the University of Cagliari, Italy, in 2006 and the Ph.D. degree in Electronics, Telecommunications and Information Technologies Engineering from the University of Bologna, Italy, in 2010. He has been a postdoctoral Research Fellow at ETH Zürich. He currently is an Assistant Professor at the University of Bologna, Department of Computer Science and Engineering (DISI). His research interests concern parallel programming model and architecture design in the single-chip multiprocessors domain, with special emphasis on compilation for heterogeneous architectures, predictable execution models, efficient usage of on-chip memory hierarchies and SoC virtualization. He has published more than 80 papers in peer reviewed international journals and conferences.

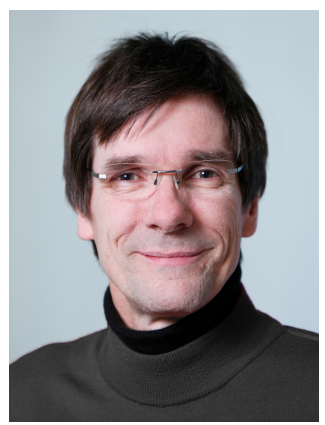

Lothar Thiele joined ETH Zürich, Switzerland, as a full Professor of Computer Engineering, in 1994. His research interests include models, methods and software tools for the design of embedded systems, cyberphysical systems, sensor networks, embedded software and bioinspired optimization techniques. Lothar Thiele is associate editor of INTEGRATION - the VLSI Journal, Journal of Signal Processing Systems, IEEE Transaction on Industrial Informatics, Journal of Systems Architecture, IEEE Transactions on Evolutionary Computation, Journal of Real-Time Systems, ACM Transactions on Sensor Networks, and ACM Transactions on Cyberphysical Systems. In 1986 he received the "Dissertation Award" of the Technical University of Munich, in 1987, the "Outstanding Young Author Award" of the IEEE Circuits and Systems Society, in 1988, the Browder J. Thompson Memorial Award of the IEEE, and in 2000-2001, the "IBM Faculty Partnership Award". In 2004, he joined the German Academy of Sciences Leopoldina. In 2005, he was the recipient of the Honorary Blaise Pascal Chair of University Leiden, The Netherlands. Since 2009 he is a member of the Foundation Board of Hasler Foundation, Switzerland. Since 2010, he is a member of the Academia Europaea. In 2013, he joined the National Research Council of the Swiss National Science Foundation. Lothar Thiele received the "EDAA Lifetime Achievement Award" in 2015.

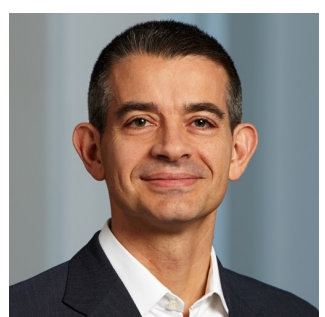

Luca Benini holds the chair of digital Circuits and systems at ETHZ and is Full Professor at the Università di Bologna. Dr. Benini's research interests are in energy-efficient system design for embedded and high-performance computing. He is also active in the area of energy-efficient smart sensors and ultra-low power VLSI design. He has published more than 800 papers, five books and several book chapters. He is a Fellow of the IEEE and the $\mathrm{ACM}$ and a member of the Academia Europaea. He is the recipient of the 2016 IEEE CAS Mac Van Valkenburg award. 